\title{
Developmental and Interventional Plasticity of Motor Maps after Perinatal Stroke
}

\author{
Sarah Y. Zhang, ${ }^{1}{ }^{\circledR}$ Matthew S. Jeffers, ${ }^{1}$ Diane C. Lagace, ${ }^{1,2,3}$ Adam Kirton, ${ }^{4,5,6,7,8}$ and ${ }^{\circledR}$ Gergely Silasi ${ }^{1,3}$ \\ ${ }^{1}$ Department of Cellular and Molecular Medicine, Faculty of Medicine, University of Ottawa, Ottawa, Ontario, Canada K1H 8M5, ${ }^{2}$ Ottawa Hospital \\ Research Institute, Neuroscience Program, Ottawa, Ontario, Canada K1H 8L6, ${ }^{3}$ Brain and Mind Research Institute, University of Ottawa, Ottawa, \\ Ontario, Canada K1H 8M5, ${ }^{4}$ Alberta Children's Hospital, Calgary Pediatric Stroke Program, Calgary, Alberta, Canada K1H 8M5, ${ }^{5}$ Alberta Children's \\ Hospital Research Institute, Calgary, Alberta, Canada T2N 1N4, ${ }^{6}$ Hotchkiss Brain Institute, Calgary, Alberta, Canada T2N 4N1, ${ }^{7}$ Department of \\ Pediatrics, Cumming School of Medicine, University of Calgary, Calgary, Alberta, Canada T2N 4N1, and ${ }^{8}$ Department of Clinical Neurosciences, \\ Cumming School of Medicine, University of Calgary, Calgary, Alberta, Canada T2N 4N1
}

Within the perinatal stroke field, there is a need to establish preclinical models where putative biomarkers for motor function can be examined. In a mouse model of perinatal stroke, we evaluated motor map size and movement latency following optogenetic cortical stimulation against three factors of post-stroke biomarker utility: (1) correlation to chronic impairment on a behavioral test battery; (2) amenability to change using a skilled motor training paradigm; and (3) ability to distinguish individuals with potential to respond well to training. Thy1-ChR2-YFP mice received a photothrombotic stroke at postnatal day 7 and were evaluated on a battery of motor tests between days 59 and 70 . Following a cranial window implant, mice underwent longitudinal optogenetic motor mapping both before and after 3 weeks of skilled forelimb training. Map size and movement latency of both hemispheres were positively correlated with impaired spontaneous forelimb use, whereas only ipsilesional hemisphere map size was correlated with performance in skilled reaching. Map size and movement latency did not show groupwise changes with training; however, mice with the smallest pretraining map sizes and worst impairments demonstrated the greatest expansion of map size in response to skilled forelimb training. Overall, motor map size showed utility as a potential biomarker for impairment and training-induced modulation in specific individuals. Future assessment of the predictive capacity of post-stroke motor representations for behavioral outcome in animal models opens the possibility of dissecting how plasticity mechanisms contribute to recovery following perinatal stroke.

Key words: behavior; motor cortex; mouse; optogenetics; reaching; recovery

Significance Statement

We investigated the utility of two cortical motor representation measures (motor map size and movement onset latency) as potential biomarkers for post-stroke motor recovery in a mouse model of perinatal stroke. Both motor map size and movement latency were associated with functional recovery after perinatal stroke, with map size showing an additional association between training responsiveness and severity of impairment. Overall, both motor map size and movement onset latency show potential as neurophysiological correlates of recovery. As such, future studies of perinatal stroke rehabilitation and neuromodulation should include these measures to help explain neurophysiological changes that might be occurring in response to treatment.

\footnotetext{
Received Dec. 18, 2020; revised Apr. 14, 2021; accepted May 20, 2021.

Author contributions: S.Y.Z., D.C.L., A.K., and G.S. designed research; S.Y.Z. and G.S. performed research; S.Y.Z. and G.S. contributed unpublished reagents/analytic tools; S.Y.Z. and M.S.J. analyzed data; S.Y.Z. and M.S.J. wrote the first draft of the paper; S.Y.Z., M.S.J., D.C.L., A.K., and G.S. edited the paper

This work was supported by Canadian Partnership for Stroke Recovery to G.S., A.K., and D.C.L.; Canadian Foundation for Innovation to G.S.; Canadian Institute for Health Research Graduate Scholarship to S.Y.Z.; and Ontario Graduate Scholarship S.Y.Z. We thank Dr. Mariana Gomez-Smith and Isabelle Sinclair-Takoff for work on model development.

The authors declare no competing financial interests.

Correspondence should be addressed to Gergely Silasi at gsilasi@uottawa.ca.

https://doi.org/10.1523/JNEUROSCI.3185-20.2021

Copyright (C) 2021 the authors
}

\section{Introduction}

Perinatal stroke occurs in 1 in every 1100 births in Canada, and is one of the leading causes of unilateral cerebral palsy and lifelong disability in children (Dunbar et al., 2020). The identification of neural biomarkers and targets for neuromodulation of perinatal stroke recovery has generated significant interest to not only predict an individual's potential for recovery, but also optimize functional gains through personalized interventions and providing measures for responsiveness to therapy (Bernhardt et al., 2016; Boyd et al., 2017; Stinear et al., 2019; Dukelow and Kirton, 2020). Unfortunately, predictors for this population are 


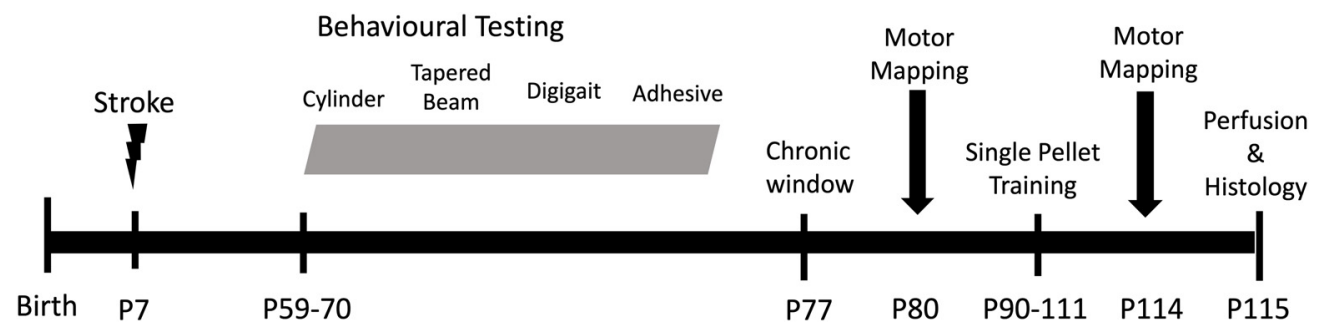

Figure 1. Experimental timeline. All time points are depicted as days since birth (P1).

poorly defined. Mirror movements (Riddell et al., 2019) and spectroscopic markers of brain metabolism (Carlson et al., 2017) have been proposed as biomarkers for motor recovery; however, motor-evoked potentials (MEPs) evoked by transcranial magnetic stimulation (TMS) consistently show the strongest relationship to functional outcome (Eyre et al., 2007; Zewdie et al., 2017; Kowalski et al., 2019). Whether there is a similar relationship between MEPs and behavioral impairment in preclinical models of perinatal stroke remains less clear but is important to define to elucidate the mechanistic underpinnings of this relationship to enhance recovery.

Preclinical models can mimic TMS and MEP measures using intracortical microstimulation (ICMS), but with greater resolution so as to sample multiple movement sites. Such studies have shown that disruption of the cortical motor map impaired skilled movement following adult stroke (Kim et al., 2015, 2018; Touvykine et al., 2016). In perinatal animals, localized motor map disruptions dually ablate distal motor map areas and impair motor performance (Kolb and Holmes, 1983; Williams et al., 2006; Monfils et al., 2008). Additionally, unilateral ablations in perinatal animals, but not adults, have also shown increased aberrant contralesional corticospinal involvement (Castro, 1975; Kartje-Tillotson et al., 1985). However, the longitudinal effects on the motor maps remain unknown since ICMS is an invasive method that requires terminal experiments that complicate measurement of longitudinal changes in motor map responses within animals (Lim et al., 2013).

Optogenetic stimulation (Ayling et al., 2009; Hira et al., 2009; Silasi et al., 2013) can overcome the low spatial resolution of TMS techniques (Vahabzadeh-Hagh et al., 2012; Boonzaier et al., 2018), while also enabling the investigation of similar MEP parameters in mice to those that are predictive of functional outcome in human stroke, such as cortical motor map area (Koski et al., 2004) and motor conduction time (Heald et al., 1993). Additionally, optogenetics allows multi-time point and bihemispheric sampling through transcranial stimulation (Silasi et al., 2016), thus eliminating the potential for inadvertent brain damage during cranial window preparation or mapping. Multi-time point sampling can be used to measure how a rehabilitation strategy, such as skilled motor training, can change motor maps and how these changes relate to functional outcome within the same animal, which is lacking in the preclinical literature for perinatal stroke. Conversely, bihemispheric motor maps allow for the investigation of both contralateral and ipsilateral motor pathways in relation to functional outcome of the contralesional limb, as previously implicated in recovery from perinatal stroke (Carr et al., 1993; Eyre et al., 2001; Staudt, 2007; Zewdie et al., 2017).

The present study uses optogenetics-based motor mapping in a mouse perinatal stroke model to address the following experimental questions: (1) Are motor maps correlated with adult impairment? (2) Can the maps be altered by skilled motor training? (3) Does the initial behavioral impairment modulate the degree to which these metrics can change in response to training? If either motor map size or motor response latency fulfills these criteria, it suggests the suitability of these outcomes as potential biomarkers as they are sensitive to stroke-induced impairments, amenable to training-induced changes, and can stratify animals that may have a greater potential to respond to motor training. This would warrant further investigation in preclinical development of prospective perinatal stroke rehabilitation or neuromodulation therapies, which are already entering Phase 3 trials in humans (Hilderley et al., 2019).

\section{Materials and Methods}

Experimental design

Male and female Thy1-ChR2-YFP mice ( $N=39 ; 18$ male, 21 female; $B 6$. Cg-Tg(Thy1-COP4/EYFP)18Gfng/J; 007612, The Jackson Laboratory) were bred, housed, and tested in a $12 \mathrm{~h} \mathrm{light} / 12 \mathrm{~h}$ dark cycle. On postnatal $(\mathrm{P})$ day 7, 39 mice were randomly assigned to a stroke group $(n=20)$ that received a photothrombotic induction of stroke in the primary motor cortex (M1), or the control sham group $(n=19)$. The mice were weaned, and as adults completed a battery of sensorimotor behavioral tests from P59 to P70 (Fig. 1). Transcranial chronic windows were surgically implanted at P77, followed by optogenetic motor mapping sessions at two time points: pre- (P80) and post-training (P114). Single-pellet reach training was conducted at P90-P111 (Fig. 1). Sample sizes for single-pellet-related measures were $n=28$ of 39 , because of exclusion of animals that failed to acquire skilled reaching behaviors $(n=8 ; 5$ stroke, 3 sham) and animals that died unexpectedly before the final mapping session $(n=3)$. Excluded animals did not engage in reaching behaviors (i.e., forelimb did not reach through the slot) within the 3 weeks of training. All experimental procedures were conducted in accordance with the guidelines of the Canadian Council on Animal Care. Experimental protocols were approved by the University of Ottawa Animal Care Committee.

\section{Stroke induction}

On P7 (day of birth was P1), mouse pups were briefly separated (1-2 h) from the dam to undergo photothrombotic surgery (Maxwell and Dyck, 2005). We chose to induce stroke on P7, as it is roughly equivalent to human development at the time of birth. Specifically, there are similarities in the degree of cortical and striatal myelination, neuronal proliferation, and synaptic density (Hagberg et al., 1997; Clancy et al., 2001; Cavarsan et al., 2019). Additionally, the motor tracts have descended caudally by P7, but similarly to the neonatal human, the organization is primarily bilateral (Joosten et al., 1987; Terashima, 1995; Gianino et al., 1999; Hsu et al., 2006). This allows the potential for post-stroke contralesional hemispheric rewiring, as has been previously demonstrated in the clinical perinatal stroke population (Carr et al., 1993; Eyre et al., 2001). Finally, previous studies have shown feasibility for inducing photothrombosis in mice on P7 (Maxwell and Dyck, 2005; Brima et al., 2013).

Animals were anesthetized with inhalation of isoflurane (induction $4 \%$, maintained at $2 \% ; \mathrm{O}_{2}: 0.5 \mathrm{~L} / \mathrm{min}$ ), placed on a warming blanket $\left(37^{\circ}\right.$ C), and received a small midline scalp incision. A green laser $(532 \mathrm{~nm}$, $1.5 \mathrm{~mm}$ diameter, $20 \mathrm{~mW}$ power) was positioned $5 \mathrm{~cm}$ above the skull and aligned to M1 in the right hemisphere using stereotaxic coordinates 


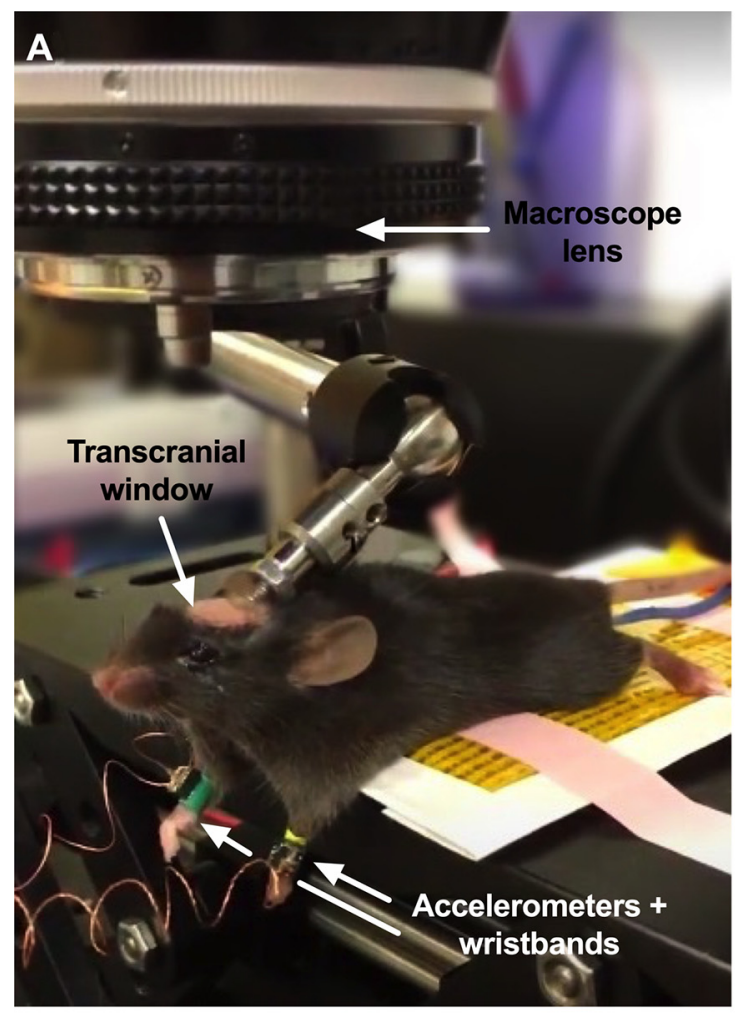

\section{B Transcranial Window}
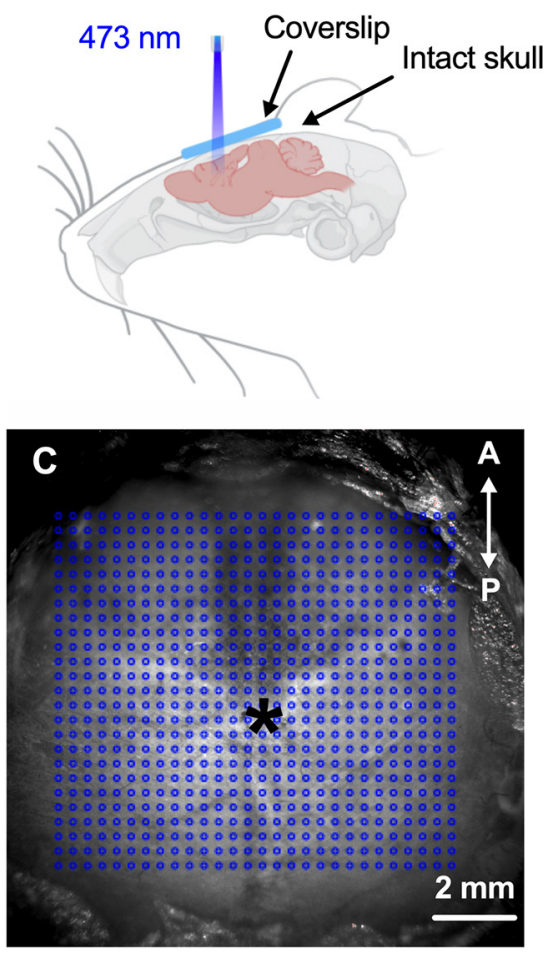

D
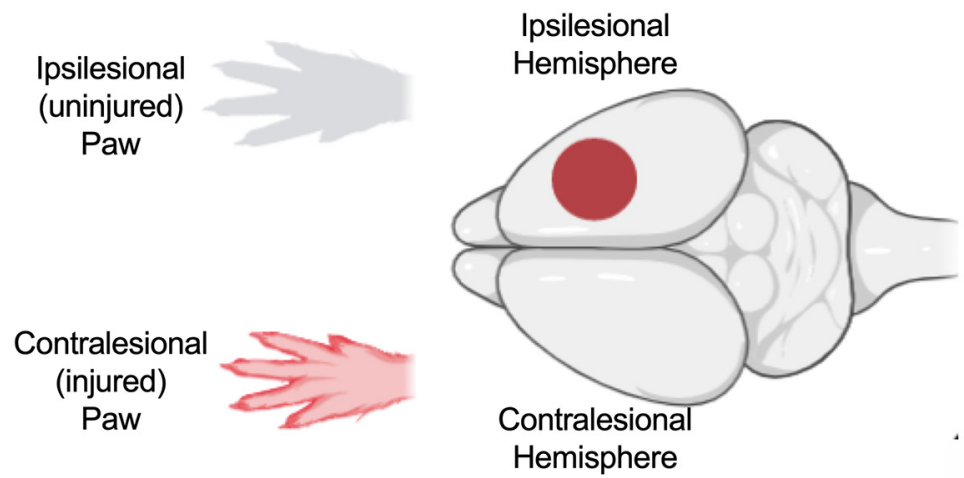

Figure 2. A, Animals were anesthetized and head-fixed underneath the stereoscope lens/optogenetic rig. Limbs were allowed to hang freely, and wrists were affixed with wristbands attached to accelerometers. $\boldsymbol{B}$, Diagram of the intact-skull transcranial window implant in the sagittal plane. $\boldsymbol{C}$, Representative image of laser stimulation matrix used to generate bihemispheric motor maps. A, Anterior; P, posterior. *Bregma. D, Schematic of the injured/uninjured hemispheres and forelimbs. (reated with BioRender (https://biorender.com/).

relative to bregma (coordinates: AP: $1.5 \mathrm{~mm}$; ML: $1.0 \mathrm{~mm}$ ). Craniotomy before illumination was not necessary as the P7 skull is sufficiently translucent to pass green light. Mice were injected with Rose Bengal dye (0.1 $\mathrm{ml} / 10 \mathrm{~g}$ body weight i.p. injection). After allowing $2 \mathrm{~min}$ for the dye to reach the brain, the laser was turned on for $6 \mathrm{~min}$. Sham animals underwent identical surgical procedures, with laser illumination preceding Rose Bengal injection. Although sham animals did not receive a photothrombotic stroke, for ease of interpretation, the right and left hemispheres are hereafter referred to in all animals (sham and stroke) as the ipsilesional and contralesional hemispheres, respectively (Fig. 2D). After photothrombosis, the incision was closed with horizontal mattress sutures using 3-0 silk thread, and pups were returned to the dams.

\section{Behavior testing}

Cylinder. Mice were placed in a clear, hollow, Plexiglas cylinder (10 cm diameter, $15 \mathrm{~cm}$ height) and allowed to explore the cylinder wall until 25 rears occurred (Schallert et al., 2000; Fleming et al., 2004). The placement of paws on the cylinder wall during rearing was filmed from below using a Raspberry Pi camera (Raspberry Pi Foundation).
Contralesional paw preference during rearing behavior was quantified with the following formula:

$$
\text { Contralesional Paw Preference }=\frac{\text { contralesional }+\frac{1}{2} \text { bilateral }}{\text { total }} \times 100 \%
$$

where contralesional is the number of times the animal used the contralesional paw to contact the cylinder wall, bilateral is the number of times both paws were used simultaneously, and total is the number of times the mouse contacted the cylinder wall with any paw while rearing.

Beam. A tapered beam $(100 \mathrm{~cm}$ length, $3.5-0.5 \mathrm{~cm}$ width) with a $1.0 \mathrm{~cm}$ ledge was used to quantify paw placement accuracy during walking (Schallert et al., 2002; Fleming et al., 2004). Whenever the mouse stepped off the main level onto the depressed ledges, a foot fault was recorded. The beam was marked in $1 \mathrm{~cm}$ intervals so that the distance of the first foot fault could also be recorded. The mice were trained/tested on the task for three trials a day over $5 \mathrm{~d}$. An 
individual trial was defined as a traversal of the full length of the beam. Beam performance was filmed with a wide-angle Raspberry Pi camera and analyzed for number of foot faults and distance to first foot fault on days 4 and 5 .

Adhesive removal. The adhesive removal test was used to detect sensorimotor asymmetries (Bouet et al., 2009). A small piece of adhesive tape $\left(0.5 \mathrm{~cm}^{2}\right)$ was adhered on glabrous skin in the center of both forepaws. The mice were then placed in a Plexiglas cylinder $(10 \mathrm{~cm}$ diameter, $15 \mathrm{~cm}$ height) and filmed until both pieces of tape were removed from the forepaws, or until the $2 \mathrm{~min}$ time limit was reached. The latency to contact the forepaw with the mouth and remove the piece of tape from the paw was determined from video recordings. If the adhesive tape was not contacted or removed within the allotted time limit, then the measure was recorded as $120 \mathrm{~s}$. All measures were averaged across $5 \mathrm{~d}$ of testing.

Digigait. The Digigait treadmill (Mouse Specifics) was used to measure gait parameters during locomotion (Hampton et al., 2004). Animals ran on the treadmill at a speed of $20 \mathrm{~cm} / \mathrm{s}, 8^{\circ}$ incline until a minimum of $3 \mathrm{~s}$ of steady and consistent gait was obtained. Videos were then analyzed with Digigait software, which automatically identifies paw footprints and calculates kinematics for 43 different gait parameters. However, not all 43 gait parameters were deemed to be clinically relevant to perinatal stroke. Therefore, we chose to analyze a subset of the measures that have been previously shown to be affected after perinatal stroke, including the following: (1) swing time (i.e., the amount of time the limb is in the aerial phase of the stride cycle) and \%swing/stride (i.e., the proportion of the stride cycle in which the limb is in the swing phase) (Wang and Wang, 2012); (2) propel time (i.e., the amount of time the limb is propelling into the next step) and \%propel/stride (Zwick et al., 2001); (3) stance time (i.e., the amount of time the limb is spent standing), and associated measures of \%stance/stride and stance/swing ratio (i.e., the ratio between the stance and swing durations) (Wang and Wang, 2012); (4) stride duration, or the amount of time required for a limb to undergo a full stride cycle (Wang and Wang, 2012); (5) stride length (the equivalent of a step length in humans) (Bell et al., 2002); (6) stride frequency (or cadence) (Bell et al., 2002; Wang and Wang, 2012); and (7) paw angle (i.e., the degree of external rotation of the paw during a step) and associated measure of paw angle variability (Wren et al., 2005; Rethlefsen and Kay, 2013).

Single-pellet reaching. The single-pellet reaching task was dually used as a quantitative measure of skilled forelimb function and motor skill training (Farr and Whishaw, 2002). Mice were placed in Plexiglas testing boxes ( $19.5 \mathrm{~cm}$ length, $8 \mathrm{~cm}$ width, $20 \mathrm{~cm}$ height) and underwent daily training to reach and grasp a millet seed pellet through a $1-\mathrm{cm}$-wide slot with the contralesional limb. The training period lasted 3 weeks $(6 \mathrm{~d} /$ wk), where sessions involved 10 min of reaching or a maximum of 30 trials, whichever was achieved first. A trial was defined as the period of time encompassing all movements of the forelimb through the reaching slot from the initial placement of a millet seed until that seed had either been displaced (through knocking it away or retrieval) or the mouse abandoned the seed by moving toward the back wall of the testing box. Mice were food restricted during training ( $80 \%$ pretraining weight) to encourage reaching. Mice were filmed using a Raspberry Pi camera and scored on all days of training; however, the final $3 \mathrm{~d}$ of testing were also pooled to calculate the following metrics of post-training performance as follows:

Overall success rate was calculated as follows:

$$
\text { Overall Success Rate }=\frac{\text { successful trials }}{\text { successful }+ \text { unsuccessful trials }} \times 100 \%
$$

Where successful trials were those where the mouse successfully grasped the pellet and brought it through the slot to its mouth (regardless of the number of forelimb extensions required to do so). Unsuccessful trials were those where the mouse displaced the pellet from the well in which it was placed without retrieving it, or where the mouse made attempts to retrieve the pellet without displacing it and abandoned the trial by moving to the back of the testing chamber with the pellet still in place.

Average number of reach attempts was calculated as follows:

$$
\text { attempts } / \text { success }=\frac{\sum(\text { reach attempts to successfully complete trial })}{\text { successful trials }}
$$

Where a reach attempt was defined as any extension of the arm through the reaching slot during a trial. All animals that engaged with the task (i.e., those that successfully acquired the reaching behavior) were able to achieve at least one successful trial by the last $3 \mathrm{~d}$.

First-attempt success rate was calculated as follows:

$$
\begin{gathered}
\text { First }- \text { attempt Success Rate } \\
=\frac{\text { successful trials with only } 1 \text { reach attempt }}{\text { successful }+ \text { unsuccessful trials }} \times 100 \%
\end{gathered}
$$

Each metric provided unique information regarding how mice in each group performed the single-pellet task. Overall success rate provided a measure of ability to eventually retrieve the pellet, regardless of the effort required. This is contrasted by first-attempt success rate, which is a measure of ability to retrieve the pellet with a single, high-accuracy, movement. Average number of reach attempts is related to first-attempt success rate (as an animal with a high number of average attempts must necessarily have a lower first-attempt success rate) but provides additional information regarding the amount of effort required for the mouse to achieve their "overall success rate."

\section{Chronic window implantation}

Chronic transcranial windows were prepared as previously described (Silasi et al., 2016). Briefly, animals were anesthetized, injected with meloxicam (5 mg/kg, s.c.), and the surgical area cleaned. An incision along the midline of the scalp was made, and the skin was cut away to expose the surface of the skull from $\lambda$ to $\sim 3 \mathrm{~mm}$ anterior to bregma. The skull was then cleaned of hair and fascia and affixed with a circular glass coverslip (Ted Parker; product \#260368, $10 \mathrm{~mm}$ diameter) using clear-drying dental cement (Parkell; product: C\&B Metabond, SKU: S380) (Fig. 2B). A small screw (McMaster-Carr; product \#94355A216) for head fixation during cortical stimulation procedures was then placed on the surface of the skull with additional dental cement (AP: $-5.0 \mathrm{~mm}$; ML: $0.0 \mathrm{~mm}$ ).

\section{Motor mapping}

Following chronic window implantation, mice underwent two sessions of cortical motor mapping, as previously described (Silasi et al., 2013). For each session, mice were anesthetized with a subcutaneous injection of a ketamine $(75 \mathrm{mg} / \mathrm{kg})$ and xylazine $(4 \mathrm{mg} / \mathrm{kg})$ cocktail. Top-ups of the cocktail (ketamine: $35 \mathrm{mg} / \mathrm{kg}$; xylazine: $2 \mathrm{mg} / \mathrm{kg}$, s.c.) were provided as needed. Animal body temperature was maintained at $37^{\circ} \mathrm{C}$ with a homeothermic heating pad. Mice were affixed to an automated motor mapping macroscope (LabeoTech) that directed a $50 \mu \mathrm{m}$ beam of $473 \mathrm{~nm}$ laser light to individual points across both hemispheres in a random grid pattern. Forelimb movements were recorded with accelerometers affixed to the wrists of both forelimbs (Fig. $2 A$ ). The minimum laser power required to evoke a limb movement (i.e., resting motor threshold [RMT]) was first obtained in the "hotspot" of the motor maps, where there was greatest sensitivity to light stimulation. In the sham animals, this was achieved by sampling both hemispheres by manually stimulating a $0.5 \times 0.5 \mathrm{~mm}$ area around the coordinates AP: $0.5 \mathrm{~mm}, \mathrm{ML}: \pm 1.0$ $\mathrm{mm}$ relative to bregma. In the stroke animals, the RMT was established only in the uninjured hemispheres, as perilesional tissue was often unresponsive. The site of largest evoked movement was determined from this reduced map, and thresholding was calculated relative to this point using the following procedure. Laser duration was set to $5 \mathrm{~ms}$, and laser power was increased at 5\%-10\% intervals (starting at 20\% maximum power; $12.5 \mathrm{~mW}$ ) until movement was visually observed in the real-time accelerometer voltage trace. Once movement was consistently obtained, laser power was subsequently decreased by $2 \%$ intervals until the movement disappeared, and the RMT was set at the last interval that evoked movement. To collect the full maps, a matrix of stimulation points was selected over the cortical surface as shown in Figure 2C (300 $\mu \mathrm{m}$ spacing 


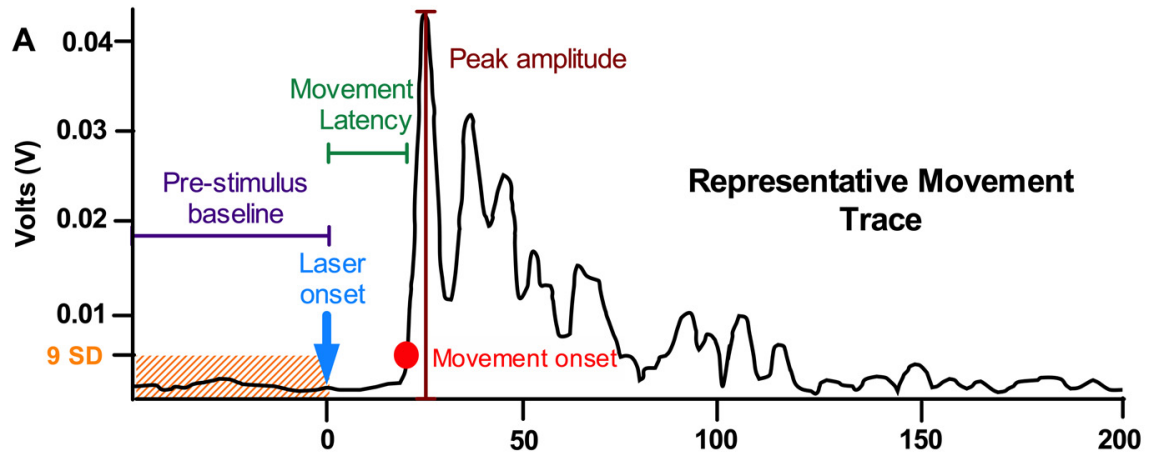

Latency Maps

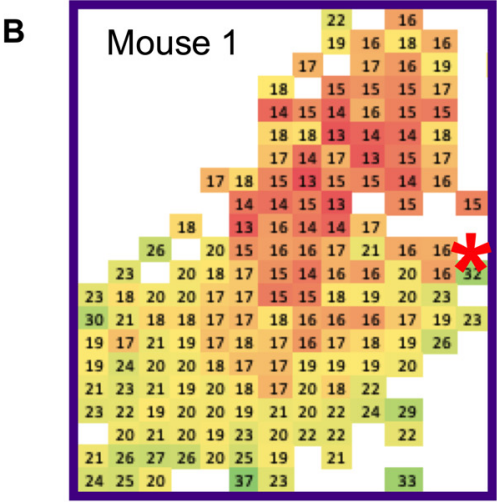

Time (ms)
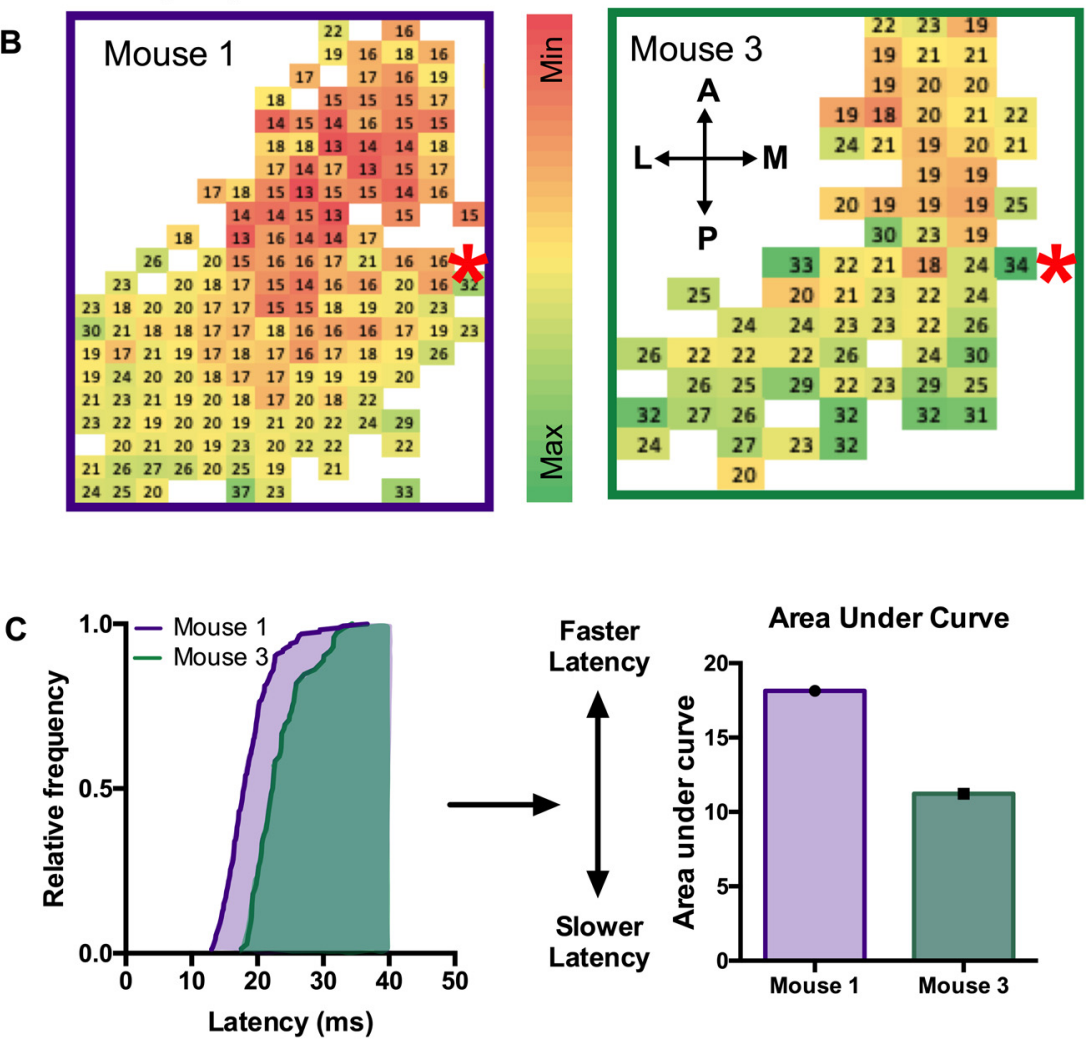

Figure 3. A, Prestimulus baseline movement (purple) was collected $100 \mathrm{~ms}$ before laser onset (blue arrow). Following laser stimulation, only movements with amplitudes which (1) occurred within $250 \mathrm{~ms}$ following laser onset and (2) exceeded 9 SDs of baseline movement amplitude (orange box) were included. Movement latency (green) refers to the amount of time elapsed for a movement to reach the 9 SD threshold, and peak amplitude (maroon) refers to the maximum accelerometer deflection. $B$, Diagram showing raw latency maps from the left hemisphere of 2 representative sham animals, where each colored cell represents a movement site, and the numbers represent latencies. Latency maps were converted to cumulative distributions in $\mathbf{C}$. Individual maps are matched to corresponding traces based on color. Red asterisk indicates bregma. A, Anterior; $\mathrm{P}$, posterior; M, medial; L, lateral. C, Diagram represents calculation (left) and interpretation (right) of AUC measurements cumulative latency distributions from 2 representative sham animals in $\boldsymbol{B}$.

between each point extending from $\sim \mathrm{AP} \pm 3.5 \mathrm{~mm}$ to $\sim \mathrm{ML} \pm 4.5 \mathrm{~mm}$ ) and stimulated in a random order at $150 \%$ of the RMT with an interstimulus interval of $0.5 \mathrm{~s}$. All stimulation points within an animal in both hemispheres used the same 150\% RMT value, but each animal had its own unique value for RMT. Motor maps were thus bihemispheric representations (i.e., over both the ipsilesional and contralesional hemispheres) of the contralesional (injured) limb (Fig. 2D).

Motor maps were analyzed with custom MATLAB code (The MathWorks). Evoked accelerometer signals were digitized and summed across all three axes to quantify peak movement deflection/amplitude (Fig. 3A). To eliminate breathing and movement artifacts, only responses with amplitudes that exceeded 9 times the SD of the $100 \mathrm{~ms}$ prestimulus period were considered lightevoked movements (Fig. 3A). Map size was calculated as the absolute number of active pixels (i.e., stimulation points that evoked movement). Motor response latency was determined as the amount of time between light stimulation and when a movement trace exceeded the 9 SD threshold. Maps were then thresholded to exclude movement sites with latencies $<13 \mathrm{~ms}$ and $>40 \mathrm{~ms}$ as determined from latency measurements from previous optogenetic mapping studies (Ayling et al., 2009; Hira et al., 2009; Harrison et al., 2012). Individual latencies from each hemisphere in each animal (Fig. 3B) were pooled and plotted as cumulative frequency distributions (Fig. $3 C$, left). In order to calculate the delay or reduction in motor response latencies, area under the curve (AUC) of latency distributions was calculated for each animal whereby faster motor response latency distributions corresponded to larger AUCs, and slower distributions corresponded to smaller AUCs (Fig. 3C, right). In 9 of 20 stroke animals, there were fewer than three movement sites; therefore, movement latency AUC was recorded as 0 . Post-single-pellet change in map size and movement latency AUC was calculated as the difference between the before and after single-pellet time points, then divided by the pre-single-pellet value.

\section{Euthanasia and histology}

On the day following the last cortical imaging session, animals were deeply anesthetized and transcardially perfused with PBS $(7 \mathrm{ml} / \mathrm{min}$ for $6 \mathrm{~min})$ and $10 \%$ neutral buffered formalin $(7$ $\mathrm{ml} / \mathrm{min}$ for $10 \mathrm{~min}$ ). Brains were removed and postfixed at $4^{\circ} \mathrm{C}$ for a minimum of $24 \mathrm{~h}$ in formalin, then cryoprotected in 30\% sucrose in PBS until saturated. Subsequently, brains were frozen at $-80^{\circ} \mathrm{C}$, then mounted and cryo-sectioned at $50 \mu \mathrm{m}$ in $-22^{\circ} \mathrm{C}$. Every second coronal section spanning from the base of the olfactory bulb (AP: $3.4 \mathrm{~mm}$ ) to the back of the hippocampus (AP: $-2.6 \mathrm{~mm}$ ) was mounted on $1 \%$ gelatin-coated slides and stained with cresyl violet.

Cresyl violet-stained slides were imaged on a flatbed scanner (Canon 9000F MKII Flatbed Scanner) at a resolution of $1200 \mathrm{dpi}$. For each brain section, the area of intact tissue of both hemispheres was manually outlined and measured using ImageJ. The total infarct volume was calculated as the difference between the hemispheric volumes as follows:

$$
\begin{gathered}
\Sigma((\text { Contralesional }- \text { Ipsilesional }) \\
\times \text { distance }+ \text { thickness })
\end{gathered}
$$

Where contralesional is the area of the contralesional hemisphere in each section, ipsilesional is the area of the ipsilesional hemisphere, distance is the distance between each section, and thickness is the thickness of each slice. Corresponding volumes were then summed across all sectioned slices.

\section{Statistical analysis}

Behavioral, histologic, and mapping data were expressed as mean \pm SEM. All analyses were performed using SPSS (IBM) and GraphPad Prism 6. 
Unpaired $t$ tests for sham versus stroke groups were used for the following analyses: (1) lesion volume, (2) cylinder test - paw preference during rearing, (3) adhesive removal test - contact and removal times for both limbs, (4) tapered beam - distance to first foot fault, (5) latency AUC, and (6) RMT. A mixed repeated-measures ANOVA was used for the remainder of the behavioral test measures (tapered beam, Digigait, and single-pellet learning curve), and motor map measures (size and pre- vs post-singlepellet analysis). Sidak-corrected $t$ tests were used for post hoc analysis with $\alpha=0.05$ to determine statistical significance. For all of the above repeated-measures ANOVAs, the between-subject factor was treatment (stroke vs sham). The within-subject factors for Digigait, tapered beam (number of foot faults), single-pellet learning curve, map size, and pre- versus post-single-pelleting reaching maps were as follows: (1) contralesional/ipsilesional side and forelimb/hindlimb, (2) contralesional/ipsilesional side, (3) days elapsed, (4) and (5) hemisphere (contralesional vs ipsilesional), and (6) time (pre vs post), respectively. Pearson's correlations were used to correlate (1) behavioral outcomes with motor map size and movement latency AUC, (2) post-single-pellet change in map size and movement latency AUC with pre-single-pellet map size and latency AUC, and (3) post-single-pellet change in map size and latency AUC with average number of reach attempts required during the single-pellet task.

\section{Results}

\section{Perinatal photothrombosis induces stroke in the motor} cortex and striatum

Induction of a photothrombotic stroke on P7 produced a mean lesion volume of $14.1 \pm 2.2 \mathrm{~mm}^{3}$ when quantified at study endpoint (when mice were mature adults, 16 weeks of age) (Fig. 4A). Visual inspection of histologic sections confirmed that even the smallest lesions extended through all cortical layers (Fig. 4B). The majority of animals ( 15 of 20 ) also displayed damage within the corpus callosum extending into the dorsal striatum (Fig. 4C).

\section{Perinatal stroke reduces motor map size and movement latency AUC in both hemispheres}

Using optogenetic stimulation of the motor cortex, we generated bihemispheric cortical motor maps for the contralesional limb from both sham and stroke animals (Fig. 5A). RMT was not significantly different between the sham and stroke groups (Table $\left.1 ; t_{(37)}=-1.021, p=0.315\right)$. Furthermore, RMT was not correlated with lesion volume or map size $(p>0.05)$. RMT was significantly negatively correlated with latency AUC in both the contralesional (Table 2; $R=-0.39, p=0.015$ ) and ipsilesional hemispheres (Table 2; $R=-0.54, p=0.0004$ ), meaning that animals that displayed higher RMTs also had a longer movement latency when stimulated.

Map size (as quantified by the number of movement sites) was reduced by $81 \%$ in the ipsilesional hemisphere of stroke animals relative to shams (Fig. $5 B$; stroke: $18.5 \pm 6.08$ pixels, sham: $99.3 \pm 8.05$ pixels). In the opposite, contralesional hemisphere, map size was reduced by $54 \%$ in the stroke group (Fig. $5 B$; stroke: $46.5 \pm 8.05$ pixels, sham: $100.2 \pm 8.23$ pixels). A two-way repeated-measures ANOVA showed a main effect of stroke on motor map size $\left(F_{(1,37)}=10.7, p=0.002\right)$. Sidak's post hoc tests further showed that this effect was maintained over both the ipsilesional $(p<0.001)$ and contralesional hemispheres $(p<0.001)$.
Additionally, within the stroke group, the map size in the ipsilesional hemisphere was significantly smaller than that of the contralesional hemisphere $(p<0.001$, Sidak's test $)$, whereas there was no difference in the sham group ( $p=0.87$, Sidak's test). A significant negative correlation between lesion volume and map size in the ipsilesional hemisphere was observed (Table 2; $R=$ $-0.47, p=0.039$ ), but not between lesion volume and latency AUC (Table 2; $R=-0.37, p=0.11$ ).

Animals with a stroke had significantly longer latencies to evoke movement, as shown by a reduction in the area under the cumulative distribution curves (AUC) (Fig. 5C; $F_{(1,37)}=4.51$, $p=0.04)$. Specifically, in the ipsilesional hemisphere, the movement latency AUC was reduced by $49 \%$ in the stroke group (Fig. $5 D$; stroke: $7.0 \pm 1.16 ; p<0.001$, sham: $13.7 \pm 1.19$, Sidak's test). In the contralesional hemisphere, the latency AUC was reduced by $26 \%$ (Fig. $5 D$; stroke: $10.1 \pm 0.82$, sham: $13.6 \pm 0.84$; $p=0.005$, Sidak's test). Within the stroke group, there was also a significant reduction in movement latency AUC in the ipsilesional versus contralesional hemisphere ( $p=0.005$, Sidak's test), whereas the sham animals showed no difference between hemispheres ( $p=0.931$, Sidak's test).

Together, these results show that P7 stroke significantly reduced cortical movement representation of the impaired limb in both hemispheres, with the majority of movement sites originating from the contralesional hemisphere. Degree of map reduction in the ipsilesional hemisphere was correlated with the size of the lesion. In addition, the latency of the evoked movements from either hemisphere was significantly longer in stroke compared with sham mice, with the contralesional hemisphere evoking the shortest latency movements of the impaired limb following stroke.

\section{Pretraining motor map size, movement latency AUC, and lesion volume correlate with spontaneous limb use and skilled reaching}

In the cylinder task (Fig. 6A), there was no significant decrease in preference for the contralesional limb in stroke animals during rearing behaviors (Fig. $6 B$; sham: $53.5 \pm 2.9 \%$, stroke: 
A
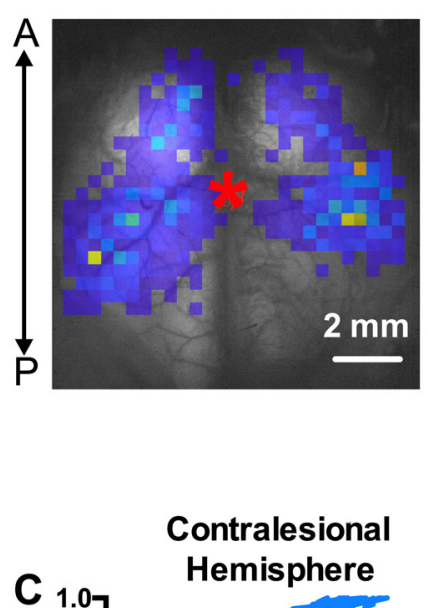

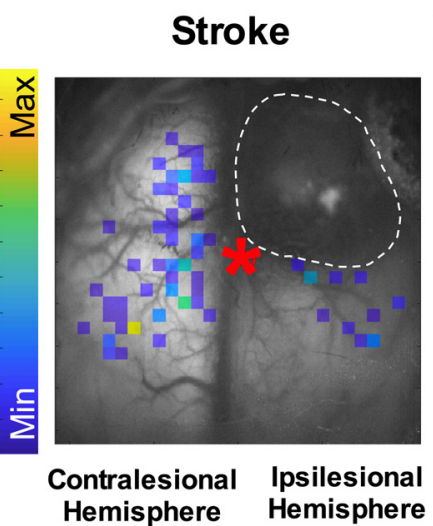

Hemisphere

psilesional
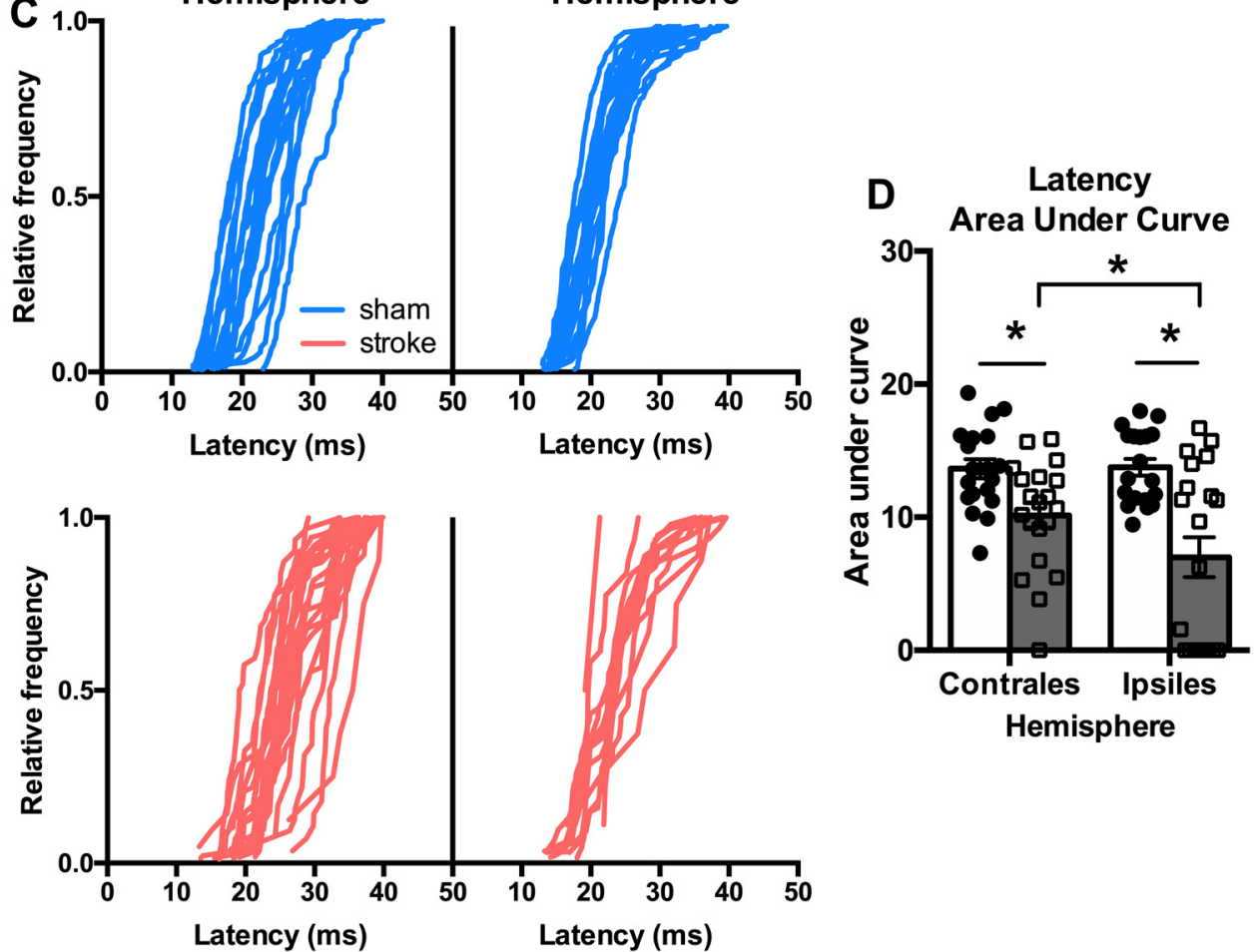

Map Size

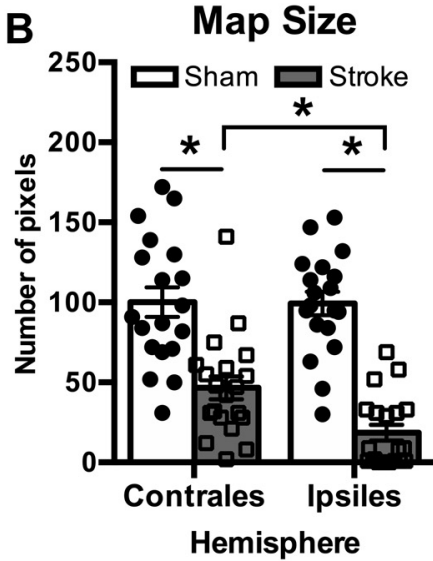

Hemisphere

Figure 5. A, Representative cortical motor maps of the contralesional forelimb from a sham (left; contralesional map size: 165 pixels, ipsilesional map size: 114 pixels) and stroke (right; contralesional map size: 47 pixels, ipsilesional map size: 10 pixels) animal. Colored pixels represent stimulation points which evoked forelimb movement, with brighter pixels representing larger movements. Red asterisk indicates bregma. A, Anterior; P, posterior. B, Contralesional forelimb map size was significantly reduced in both the contralesional and ipsilesional hemispheres in stroke (gray bars) versus sham animals (white bars). C, Cumulative frequency distributions for contralesional forelimb movement latencies in both stroke (pink lines) and sham (blue lines) animals from the contralesional (left) and ipsilesional (right) hemispheres. $\boldsymbol{D}$, Similarly, area under latency distribution curves was significantly reduced in stroke (gray bars) versus sham animals (white bars) in both hemispheres. Sham $(n=19)$, stroke $(n=20)$. Data are mean \pm SEM. ${ }^{*} p<0.05$.

$46.1 \pm 3.0 \% ; t_{(37)}=1.78, p=0.083$, unpaired $t$ test). There was a significant positive correlation between contralesional limb preference on the cylinder test and map size in the combined sham and stroke groups for both the contralesional (Fig. $6 C ; R^{2}=0.25$, $p=0.0011$ ) and ipsilesional hemisphere (Fig. $6 D ; R^{2}=0.13$, $p=0.026)$. When the sham and stroke groups were considered separately, there was a significant correlation for the sham group in the contralesional $\left(R^{2}=0.21, p=0.050\right)$, but not ipsilesional hemisphere $\left(R^{2}=0.053, p=0.34\right)$. For the stroke group alone, use of the contralesional limb and map size were not correlated in either the contralesional $\left(R^{2}=0.18, p=0.062\right)$ or ipsilesional hemispheres $\left(R^{2}=0.077, p=0.24\right)$. Similarly, movement latency AUC was positively correlated with contralesional preference in both hemispheres (Fig. 6E,F; contralesional hemisphere: $R^{2}=$
0.19, $p=0.005$, ipsilesional hemisphere: $R^{2}=0.11, p=0.037$ ). However, no significant correlations were observed in either hemisphere when the sham and stroke groups were considered separately $(p>0.050)$. Overall, these data suggest that the greater the map size and shorter the movement latency in both hemispheres, the greater the preference for the contralesional limb.

In the single-pellet reaching task (Fig. $7 A$ ), there was no significant difference between stroke versus sham in the number of successful trials on any one of the $18 \mathrm{~d}$ of training (Fig. $7 \mathrm{~B}$; $\left.F_{(1,468)}=3.20, p=0.074\right)$. There was also no difference between the sham or stroke groups in overall success rate (Fig. $7 C$; sham: $31.8 \pm 4.31 \%$, stroke: $30.0 \pm 4.56 \% ; t_{(26)}=0.283, p=0.78$, unpaired $t$ test). However, there was a significant decrease in first-attempt success rate (Fig. $7 D$; sham: $22.7 \pm 4.05$, stroke: 
Table 1. Adhesive, tapered beam, and Digigait behavioral tasks and RMT of motor maps ${ }^{a}$

\begin{tabular}{|c|c|c|c|c|c|c|}
\hline \multicolumn{2}{|c|}{ Outcome measures } & \multicolumn{2}{|c|}{ Sham } & \multicolumn{2}{|c|}{ Stroke } & $p$ \\
\hline & RMT (\%) & $20.3 \pm 1.86$ & & $23.8 \pm 2.91$ & & 0.32 \\
\hline \multirow[t]{4}{*}{ Adhesive } & Contralesional contact (s) & $22.7 \pm 4.9$ & & $27.1 \pm 4.9$ & & 0.53 \\
\hline & Contralesional removal (s) & $30.3 \pm 5.3$ & & $44.3 \pm 5.9$ & & 0.09 \\
\hline & Ipsilesional contact (s) & $25.2 \pm 5.1$ & & $25.9 \pm 5.4$ & & 0.09 \\
\hline & Ipsilesional removal (s) & $34.1 \pm 6.2$ & & $37.6 \pm 5.8$ & & 0.41 \\
\hline \multirow[t]{4}{*}{ Tapered beam } & Contralesional foot fault (no.) & $9.12 \pm 1.91$ & & $7.89 \pm 1.86$ & & 0.65 \\
\hline & Ipsilesional foot fault (no.) & $5.47 \pm 1.02$ & & $6.93 \pm 0.99$ & & 0.31 \\
\hline & Distance to first foot fault $(\mathrm{cm})$ & $47.5 \pm 4.47$ & & $41.8 \pm 4.11$ & & 0.36 \\
\hline & & Contralesional s & & Ipsilesional side & & \\
\hline \multirow{15}{*}{ Digigait } & & Sham & Stroke & Sham & Stroke & \\
\hline & Swing (s) & $0.12 \pm 0.004$ & $0.12 \pm 0.004$ & $0.11 \pm 0.003$ & $0.12 \pm 0.003$ & 0.267 \\
\hline & \% Swing/stride (ratio) & $37.45 \pm 0.59$ & $39.16 \pm 0.82$ & $36.54 \pm 0.42^{*}$ & $39.15 \pm 0.58^{*}$ & 0.001 \\
\hline & Propel (s) & $0.13 \pm 0.003$ & $0.12 \pm 0.002$ & $0.12 \pm 0.002^{*}$ & $0.11 \pm 0.003^{*}$ & 0.007 \\
\hline & $\%$ Propel/stride (ratio) & $41.56 \pm 0.94$ & $40.24 \pm 0.81$ & $40.58 \pm 0.71^{*}$ & $37.90 \pm 0.70^{*}$ & 0.007 \\
\hline & Stance (s) & $0.19 \pm 0.004$ & $0.18 \pm 0.003$ & $0.20 \pm 0.004^{*}$ & $0.18 \pm 0.003^{*}$ & 0.019 \\
\hline & $\%$ Stance/stride (ratio) & $62.55 \pm 0.59$ & $60.84 \pm 0.82$ & $63.46 \pm 0.42^{*}$ & $60.85 \pm 0.58^{*}$ & 0.001 \\
\hline & $\%$ Stance/swing (ratio) & $1.71 \pm 0.05$ & $1.59 \pm 0.05$ & $1.78 \pm 0.03^{*}$ & $1.57 \pm 0.04^{*}$ & 0.003 \\
\hline & Stride (s) & $0.31 \pm 0.01$ & $0.30 \pm 0.01$ & $0.31 \pm 0.01$ & $0.30 \pm 0.01$ & 0.416 \\
\hline & Stride length $(\mathrm{cm})$ & $6.21 \pm 0.15$ & $6.05 \pm 0.12$ & $6.20 \pm 0.13$ & $6.05 \pm 0.12$ & 0.261 \\
\hline & Stride frequency (stride/s) & $3.33 \pm 0.09$ & $3.40 \pm 0.07$ & $3.34 \pm 0.08$ & $3.39 \pm 0.07$ & 0.318 \\
\hline & & Forelimb & & Hindlimb & & \\
\hline & & Sham & Stroke & Sham & Stroke & \\
\hline & Paw angle (degrees) & $12.22 \pm 0.78$ & $12.46 \pm 0.83$ & $10.93 \pm 0.83$ & $11.38 \pm 1.00$ & 0.227 \\
\hline & Paw angle variability & $5.68 \pm 0.26^{*}$ & $6.68 \pm 0.40^{*}$ & $6.92 \pm 0.39$ & $7.52 \pm 0.41$ & 0.038 \\
\hline
\end{tabular}

${ }^{a}$ Sham $(n=19)$, stroke $(n=20)$. Data are mean \pm SEM. ${ }^{*} p<0.05$.

Table 2. Contralesional map size, latency AUC, and lesion volume correlations ${ }^{a}$

\begin{tabular}{|c|c|c|c|c|c|c|}
\hline \multirow[b]{2}{*}{ Outcome measure } & & \multicolumn{2}{|c|}{ Contralesional hemisphere $\mathrm{R}(p)$} & \multicolumn{3}{|c|}{ Ipsilesional hemisphere $\mathrm{R}(p)$} \\
\hline & & Map size (pixels) & Latency (AUC) & Map size (pixels) & Latency (AUC) & Lesion volume $\left(\mathrm{mm}^{3}\right)$ \\
\hline Cylinder & Contralesional preference (\%) & $0.50(0.0011)^{*}$ & $0.44(0.0050)^{*}$ & $0.36(0.026)^{*}$ & $0.33(0.037)^{*}$ & $-0.30(0.20)$ \\
\hline \multirow[t]{3}{*}{ Single-pellet } & Overall success rate (\%) & $-0.047(0.81)$ & $0.073(0.71)$ & $-0.10(0.62)$ & $0.11(0.57)$ & $-0.26(0.40)$ \\
\hline & First-attempt success rate (\%) & $0.013(0.95)$ & $0.13(0.50)$ & $0.22(0.27)$ & $0.26(0.18)$ & $-0.47(0.11)$ \\
\hline & Reach attempts per successful trial (no.) & $-0.20(0.32)$ & $-0.030(0.39)$ & $-0.50(0.012)^{*}$ & $-0.33(0.097)$ & $0.61(0.036)^{*}$ \\
\hline \multirow[t]{4}{*}{ Adhesive } & Contralesional contact (s) & $-0.093(0.57)$ & $-0.083(0.62)$ & $0.0075(0.96)$ & $-0.040(0.81)$ & $-0.40(0.082)$ \\
\hline & Contralesional removal (s) & $-0.21(0.20)$ & $-0.16(0.33)$ & $-0.14(0.39)$ & $-0.26(0.11)$ & $-0.32(0.17)$ \\
\hline & Ipsilesional contact (s) & $0.015(0.93)$ & $0.0087(0.96)$ & $0.070(0.67)$ & $-0.018(0.91)$ & $-0.34(0.15)$ \\
\hline & Ipsilesional removal (s) & $0.14(0.39)$ & $0.13(0.44)$ & $0.068(0.68)$ & $0.017(0.92)$ & $-0.37(0.11)$ \\
\hline \multirow[t]{3}{*}{ Tapered beam } & Contralesional foot fault (no.) & $0.15(0.36)$ & $0.11(0.52)$ & $0.29(0.070)$ & $0.16(0.33)$ & $0.34(0.14)$ \\
\hline & Ipsilesional foot fault (no.) & $0.15(0.37)$ & $0.077(0.64)$ & $0.29(0.071)$ & $0.10(0.54)$ & $-0.30(0.20)$ \\
\hline & Distance to first foot fault $(\mathrm{cm})$ & $-0.11(0.51)$ & $-0.091(0.58)$ & $-0.25(0.13)$ & $-0.048(0.77)$ & $-0.34(0.15)$ \\
\hline \multirow[t]{13}{*}{ Digigait } & Contralesional:ipsilesional & & & & & \\
\hline & Swing (s) & $0.15(0.36)$ & $0.11(0.52)$ & $0.29(0.070)$ & $0.16(0.33)$ & $-0.69(0.77)$ \\
\hline & \% Swing/stride (ratio) & $0.15(0.37)$ & $0.077(0.64)$ & $0.29(0.071)$ & $0.10(0.54)$ & $0.075(0.75)$ \\
\hline & Propel (s) & $-0.11(0.51)$ & $-0.091(0.58)$ & $-0.25(0.13)$ & $-0.048(0.77)$ & $0.29(0.22)$ \\
\hline & \% Propel/stride (ratio) & $-0.13(0.43)$ & $-0.13(0.43)$ & $-0.29(0.076)$ & $-0.12(0.48)$ & $0.34(0.14)$ \\
\hline & Stance $(s)$ & $-0.085(0.61)$ & $-0.031(0.85)$ & $-0.18(0.28)$ & $-0.0015(0.99)$ & $-0.21(0.37)$ \\
\hline & \% Stance/stride (ratio) & $-0.15(0.37)$ & $-0.10(0.53)$ & $-0.29(0.077)$ & $-0.12(0.47)$ & $-0.042(0.86)$ \\
\hline & \% Stance/swing (ratio) & $-0.19(0.24)$ & $-0.14(0.41)$ & $-0.32(0.045)^{*}$ & $-0.13(0.44)$ & $-0.071(0.77)$ \\
\hline & Stride (s) & $0.058(0.73)$ & $0.088(0.59)$ & $0.11(0.51)$ & $0.16(0.32)$ & $-0.26(0.28)$ \\
\hline & Stride length $(\mathrm{cm})$ & $0.057(0.73)$ & $0.10(0.55)$ & $0.12(0.47)$ & $0.19(0.24)$ & $-0.28(0.24)$ \\
\hline & $\begin{array}{l}\text { Stride frequency (stride/s) } \\
\text { Forelimb:hindlimb }\end{array}$ & $-0.16(0.34)$ & $-0.075(0.65)$ & $-0.21(0.20)$ & $-0.18(0.27)$ & $0.21(0.37)$ \\
\hline & Paw angle (degrees) & $-0.11(0.49)$ & $-0.065(0.69)$ & $-0.067(0.68)$ & $-0.20(0.22)$ & $0.042(0.86)$ \\
\hline & Paw angle variability & $-0.21(0.21)$ & $-0.32(0.046)^{*}$ & $-0.12(0.46)$ & $-0.35(0.031)^{*}$ & $0.035(0.88)$ \\
\hline \multirow[t]{2}{*}{ Other } & Lesion volume $\left(\mathrm{mm}^{3}\right)$ & $-0.27(0.24)$ & $0.023(0.92)$ & $-0.47(0.039)^{*}$ & $-0.37(0.11)$ & NA \\
\hline & RMT (\%) & $-0.18(0.28)$ & $-0.39(0.015)^{*}$ & $-0.19(0.25)$ & $-0.54(0.0004)^{*}$ & $0.015(0.95)$ \\
\hline
\end{tabular}


A

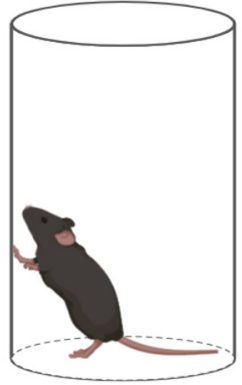

Contralesional Hemi
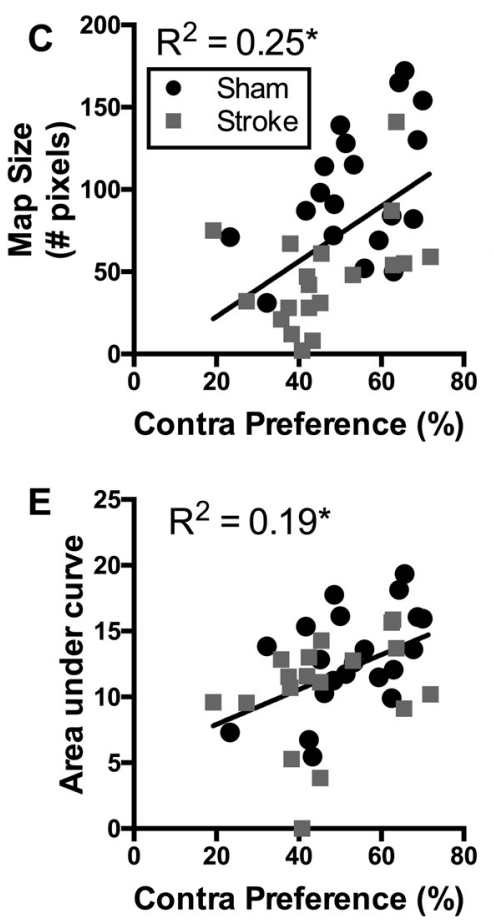

B

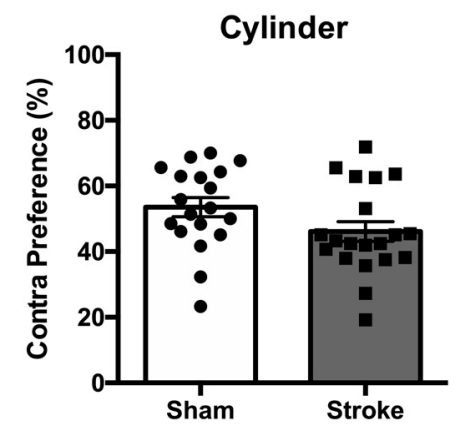

Ipsilesional Hemi
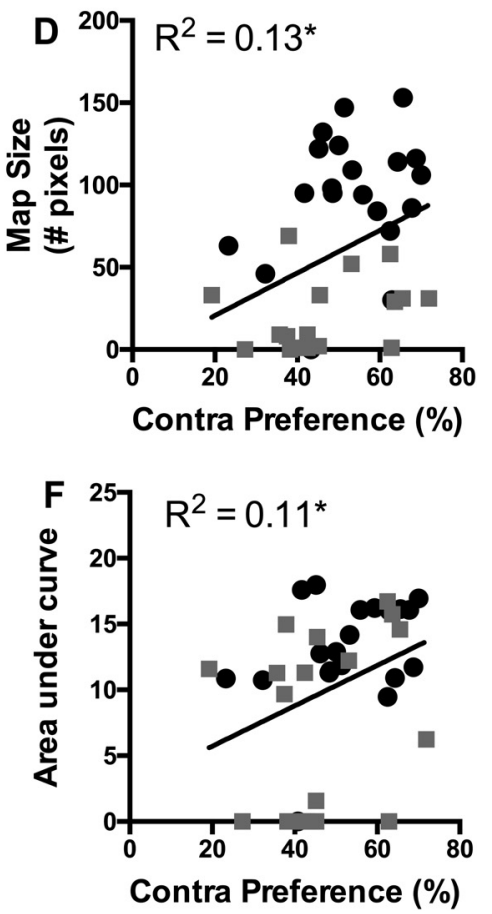

Figure 6. A, Diagram of cylinder task. B, Stroke (gray bars) was not significantly different compared with sham (white bars) in contralesional forelimb preference during rearing behavior of the cylinder task. $\mathbf{C}-\boldsymbol{F}$, Both contralesional forelimb map size and movement latency AUC were significantly correlated with contralesional forelimb use during the cylinder task in both hemispheres. Sham $(n=19)$, stroke $(n=20)$. Data are mean \pm SEM. ${ }^{*} p<0.05$. Created with BioRender (https://biorender.com/).

$11.6 \pm 3.32 ; t_{(26)}=2.08, p=0.047$, unpaired $t$ test) and a corresponding increase in the average number of reaching attempts per successful trial in the stroke animals $(2.52 \pm 0.24)$ versus sham animals $(1.68 \pm 1.36)$ (Fig. $7 E ; t_{(26)}=3.17, p=0.0040$, unpaired $t$ test). Map size and movement latency AUC were not significantly correlated with either overall or first-attempt success rate (Table 2, $p>0.05$ ). Smaller map size was significantly correlated with greater average number of reaching attempts in the ipsilesional, but not contralesional, hemisphere for the combined sham and stroke groups (Fig. $7 F, G$; contralesional hemisphere: $R^{2}=0.039, p=0.32$, ipsilesional hemisphere: $R^{2}=0.24$, $p=0.012$ ). When considered separately, neither the sham nor stroke groups showed a significant correlation between average number of reaching attempts and map size in the contralesional hemisphere $(p>0.05)$. However, although the sham group also showed no significant correlation in the ipsilesional hemisphere $\left(R^{2}=0.0069, p=0.77\right)$, the stroke group retained their significant correlation between average number of reach attempts and map size $\left(R^{2}=0.37, p=0.036\right)$. Movement latency AUC was not significantly correlated with average number of reach attempts in either hemisphere for the combined sham and stroke groups (Fig. 7H,I; contralesional hemisphere: $R^{2}=0.030, p=0.39$, ipsilesional hemisphere: $\left.R^{2}=0.11, p=0.097\right)$. Furthermore, there were no significant correlations between these variables when the sham and stroke groups were considered separately for either hemisphere $(p>0.05)$. These results show that the ipsilesional motor map size is the strongest correlate to skilled forelimb use, where the greater the map size in the ipsilesional hemisphere, the fewer number of reach attempts required to successfully complete the trial.

The remaining behavioral tasks (adhesive removal test, tapered beam) showed no significant differences between stroke versus sham animals (Table 1). In the Digigait test, sham differed from stroke animals on the ipsilesional side and in the forelimbs in a cluster of gait parameters (Table 1). However, there was no significant correlation between any measure of the adhesive removal test or tapered beam with map size and/or latency, with only 3 of 48 significant correlations in the Digigait test (Table 2). Additionally, there were no significant correlations between lesion volume and any behavioral outcome, except the average number of reach attempts per successful trial in the single-pellet task (Table 2; $R=0.61, p=0.036$ ), where the larger the lesion volume, the greater the number of reach attempts required to successfully retrieve a pellet.

\section{Skilled motor training did not result in} expansions to motor map size or reductions in motor response latency Quantification of motor map size before and after 3 weeks of single-pellet training for 30 trials per day showed that in the contralesional hemisphere, there was no significant interaction between time (pretraining vs post-training) and stroke $\left(F_{(1,26)}=1.25, p=\right.$ 0.27 , two-way repeated-measures ANOVA). Between preversus post-single-pellet training, there was no significant difference in either the sham (pre: $93.0 \pm 8.99$ pixels, post: $92.6 \pm 8.57$ pixels; $p=0.97$, Sidak's test) or stroke groups (pre: $58.2 \pm 9.65$ pixels, post: $75.2 \pm 9.20$ pixels; $p=0.148$, Sidak's test) (Fig. 8A). Before training, there was a significant reduction in contralesional hemisphere map size in the stroke compared with sham animals $(p=0.014$, Sidak's test); however, this effect was not present post-training $(p=0.179$, Sidak's test) (Fig. $8 A)$. In contrast, in the ipsilesional hemisphere, there was a significant reduction in map size in stroke versus sham at both the pre- (stroke: $27.2 \pm 7.66$, sham: $94.07 \pm 7.13, p<0.001)$ and post-single-pellet time point (stroke: $38.8 \pm 10.8$, sham: $80.6 \pm$ 10.1, $p=0.009$ ) (Fig. 8B). 
There was no significant interaction between time and stroke in movement latency AUC of the contralesional hemisphere $\left(F_{(1,26)}=0.011, p=0.92\right.$, two-way repeatedmeasures ANOVA). Moreover, there were no post hoc differences between time points in either the sham (pre: $13.5 \pm 0.76$, post: $13.5 \pm 0.71, p=0.99$, Sidak's test) or stroke animals (pre: $11.6 \pm 0.81$, post: $11.5 \pm 0.76$, $p=0.90$, Sidak's test) (Fig. $8 C$ ). In the ipsilesional hemisphere, there was no significant interaction between time and stroke $\left(F_{(1,26)}=\right.$ $0.48, p=0.49$, two-way repeated-measures ANOVA). Sidak's post hoc tests showed no significant difference between time points in either the sham (pre: $13.3 \pm 1.21$, post: $15.4 \pm 1.10, p=0.094$ ) or stroke animals (pre: $9.80 \pm 1.30$, post: $10.7 \pm 1.18, p=0.51$ ) (Fig. $8 D)$. However, there was a between-group difference in sham versus strokes at the posttraining time point $(p=0.007$, Sidak's test). Specifically, the latency of the evoked movements from the injured hemisphere after skilled training was significantly longer in stroke mice relative to shams.

Together, these data indicate that the significant loss of contralesional forelimb movement representation within the contralesional hemisphere is no longer detectable following skilled reach training. Contrastingly, latencies of evoked movements were still significantly longer in stroke animals relative to shams post-training.

Change in map size, but not movement latency AUC, is associated with motor impairment

In both the contralesional and ipsilesional hemisphere, there was a significant negative correlation between the change in map size between before and after single-pellet with the pre-single-pellet map size, with larger changes in map size being associated with smaller pre-single-pellet map size (Fig. 9A,B; contralesional: $R^{2}=0.29, p=0.0029$; ipsilesional: $\left.R^{2}=0.23, p=0.012\right)$. Similarly, in both hemispheres larger post-single-pellet change in movement latency AUC was significantly correlated with smaller pre-single-pellet movement latency AUC (Fig. 9C,D; contralesional: $R^{2}=0.42, p=0.00020$; ipsilesional: $R^{2}$ $=0.28, p=0.0070)$. These effects indicated that smaller initial map sizes and slower initial response latencies were associated with greater expansions in map size and reductions in movement latency following skilled motor training.

Change in map size was also significantly positively correlated with number of reaching attempts in both hemispheres (Fig. 9E, F; contralesional: $R^{2}=0.23, p=0.012$; ipsilesional: $R^{2}=0.38$, $p=0.0011)$. However, change in movement latency AUC was not correlated with reaching attempts in either hemisphere (Fig. 9G,H; contralesional: $R^{2}=0.069, p=0.19$; ipsilesional: $R^{2}=$ $0.021, p=0.49)$. This indicated that more severe impairment in biorender.com/).
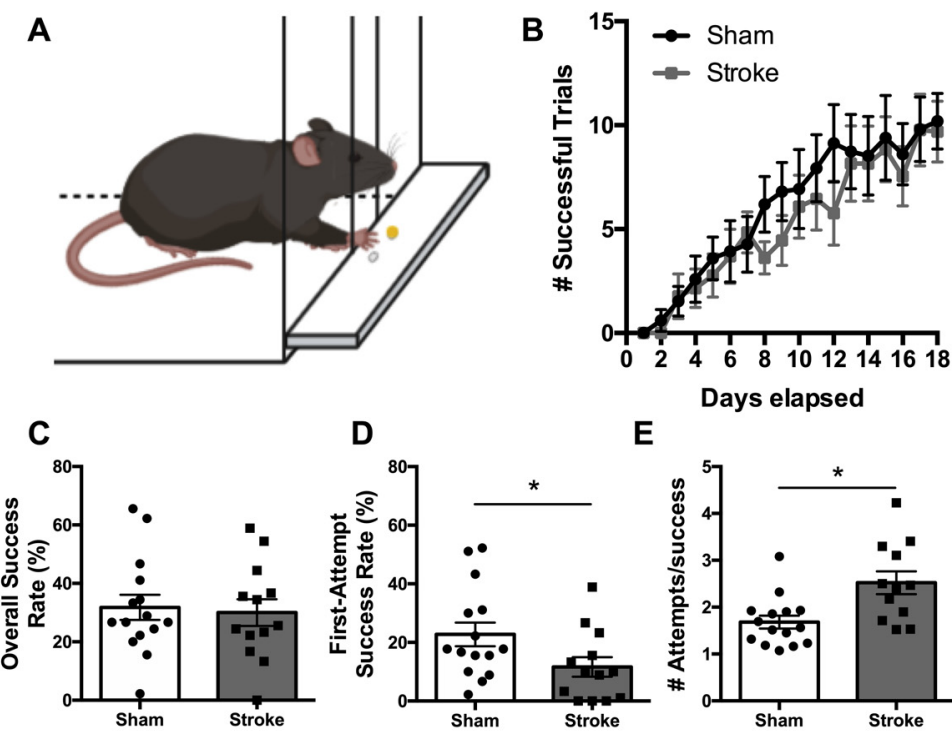

D

E
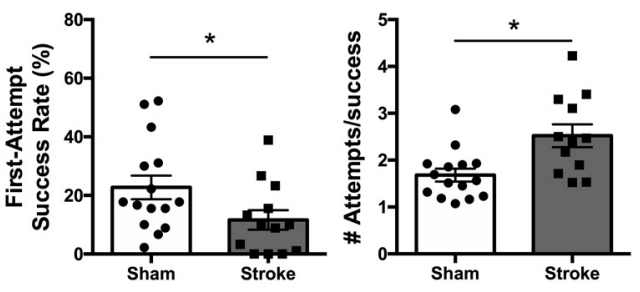

Ipsilesional Hemi

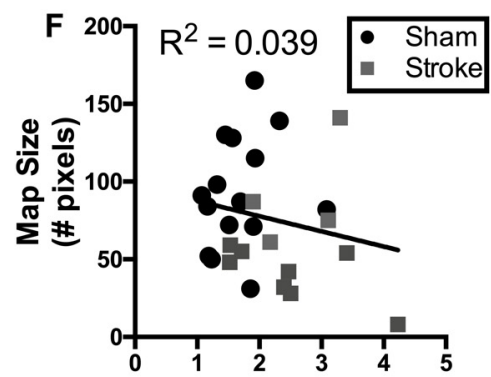

H
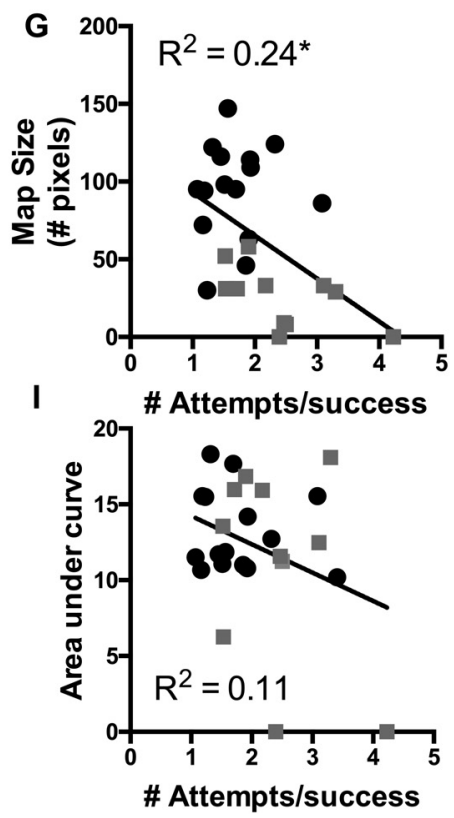

Figure 7. $\quad \boldsymbol{A}$, Diagram of the single-pellet reaching task. $\boldsymbol{B}$, Stroke animals (gray squares and line) did not differ from sham animals (black circles and line) on any day throughout the training period. C, Stroke (gray bars) and sham (white bars) achieved the same percentage of total successful trials (regardless of number of attempts). $\boldsymbol{D}$, Stroke animals achieved significantly fewer first-attempt successful trials compared with sham animals. $\boldsymbol{E}$, Stroke required a significantly greater number of reach attempts to a successful trial compared with sham. $\boldsymbol{F}, \mathbf{G}$, Map size was correlated with average number of reach attempts per successful trial in the ipsilesional, but not contralesional, hemisphere. $\boldsymbol{H}$, $\boldsymbol{I}$, Movement latency AUC was not significantly correlated in either hemisphere. Sham $(n=15)$, stroke $(n=13)$. Animals were excluded because of inability to acquire reaching behaviors. Data are mean \pm SEM. ${ }^{*} p<0.05$. Created with BioRender (https://

skilled reaching was associated with greater expansions in map size, in the absence of changes in response latency following skilled motor training.

\section{Discussion}

Perinatal stroke disrupts motor map size and motor response latency of the contralesional limb in bihemispheric motor representations

MEPs are one of the biomarkers with the strongest evidence for predicting post-stroke outcome in both adult (Stinear et al., 


\section{Contralesional Hemisphere} Ipsilesional Hemisphere

\section{Map Size}
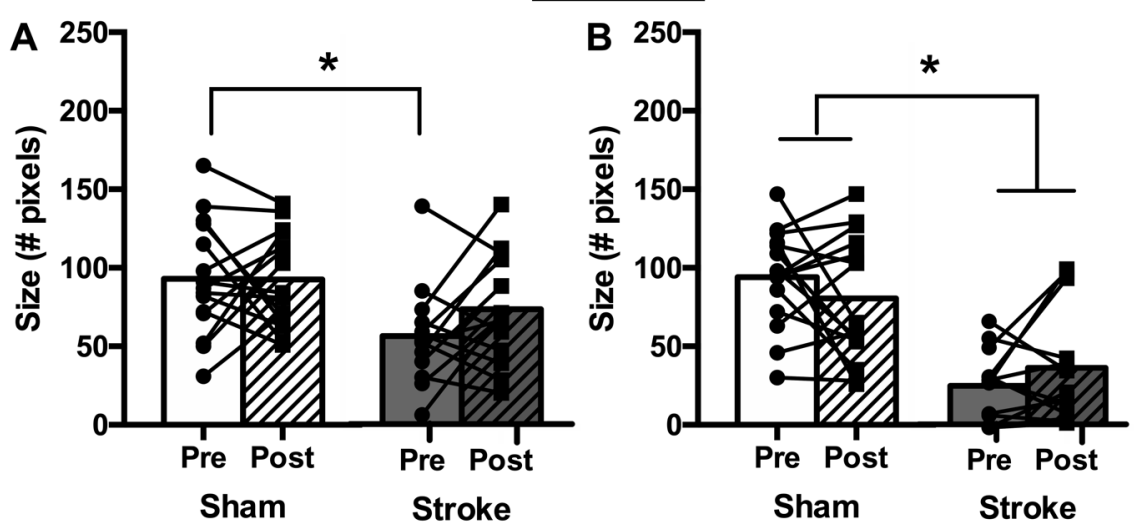

Movement Latency AUC
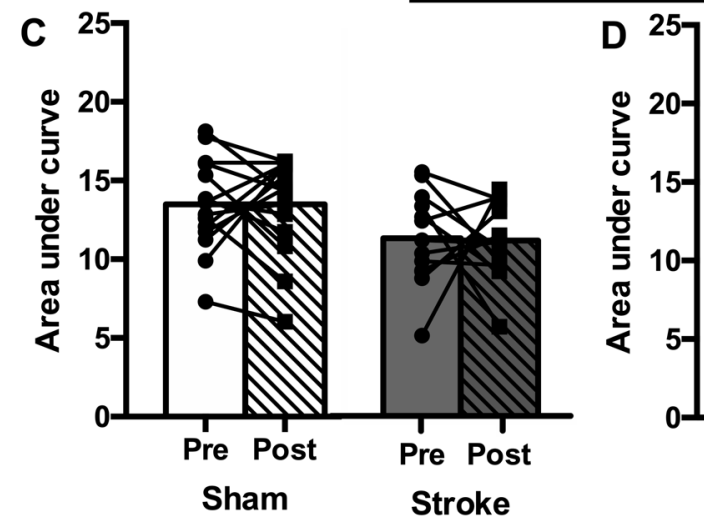

Figure 8. $\quad \boldsymbol{A}, \boldsymbol{B}$, Contralesional forelimb map size was not significantly increased following single-pellet training in either the sham (white bars) or stroke group (gray bars) in both hemispheres. $\boldsymbol{C}, \boldsymbol{D}$, There was no significant difference in movement latency AUC between time points in either hemisphere or group. Sham $(n=15)$, stroke $(n=13)$. Animals were excluded because of inability to acquire reaching behaviors. Data are reported as means, with individual animals before and after single-pellet connected by lines. ${ }^{*} p<0.05$.

2017) and perinatal stroke (Jaspers et al., 2016). However, metrics for quantifying MEPs, such as motor map size (Koski et al., 2004) or motor response latency (Heald et al., 1993; Turton et al., 1996), require further validation for use in preclinical rodent models of perinatal stroke to better understand the mechanisms behind spontaneous and training-induced recovery. We probed the utility of these potential preclinical biomarkers using suprathreshold light-based optogenetic stimulation and found similar bihemispheric motor representations to those shown previously using both optogenetics (Silasi et al., 2013) and ICMS (BrusRamer et al., 2009).

At $80 \mathrm{~d}$ after birth, we observed that unilateral stroke had decreased both ipsilesional and contralesional motor map sizes (for the stroke-affected limb), which has been previously observed for motor maps in the injured hemisphere in adult stroke (Gharbawie et al., 2005) and perinatal injury (Monfils et al., 2005). Additionally, the reduction of motor map size in the ipsilesional hemisphere was related to the size of infarct that had been induced. Second, we observed delays in motor response latency following stimulation in both the ipsilesional and contralesional hemispheres, which aligns with existing adult rodent (Anenberg et al., 2014) and human stroke findings (Turton et al., 1996; Vandermeeren et al., 2003). With both motor map size and motor response latency, we found the impairment in these metrics were more severe in the ipsilesional hemisphere than the contralesional hemisphere. As demonstrated in both animal and human studies, unilateral cortical injury before the pruning of ipsilateral descending projections can result in abnormally maintained descending corticospinal fibers to the impaired limb (Castro, 1975; Hicks and Amato, 1975; Whishaw and Kolb, 1988; Carr et al., 1993; Eyre et al., 2001). This may consequently strengthen motor control from the contralesional hemisphere more so than the ipsilesional hemisphere, whose descending corticospinal tracts are putatively reduced following cortical ablation. Detailed tracing experiments would be a worthwhile next step to offer further insight into the post-stroke rewiring of descending motor tracts. Overall, our perinatal photothrombotic stroke model had long-lasting effects in the adult brain, which impacted both motor map size and motor response latency.

It is notable that the contralesional hemisphere had such marked disruptions in both map size and motor response latency, despite the lack of direct damage. It is possible that this was because of an interruption of interhemispheric communication, leading to remote functional depression (or diaschisis) in the uninjured hemisphere. For example, one study in naive, adult rodents demonstrated a significant increase in power threshold required to elicit movement in ipsilateral motor maps following corpus callosum transection (Brus-Ramer et al., 2009). Similarly, another study using paired pulse ICMS showed strong facilitatory effects between motor maps in each hemisphere (Touvykine et al., 2020). In our study, the corpus callosum was almost completely transected at the core of the lesion in 15 of 20 mice. Our parallel loss of excitability in contralesional maps may be explained by a loss of facilitatory modulation from the ipsilesional hemisphere. Finally, the bihemispheric ablation and delays in movement latencies may stem from widespread demyelination of descending motor tracts. At the perinatal time point, oligodendrocyte progenitor cells are particularly susceptible to oxidative damage, leading to white matter damage (Dewar et al., 2003; Ferriero, 2004; McQuillen and Ferriero, 2004; Gennaro et al., 2019).

Both map size and movement latency are correlated with severity of asymmetrical limb use

One of the primary goals of the present study was to assess whether stroke-related disruption of motor map size or motor response latency was correlated with behavioral impairment in adulthood. In the cylinder task, which measures asymmetry of limb use during exploration, larger map size and faster movement latency in both the ipsilesional and contralesional hemispheres were associated with greater spontaneous use of the 
contralesional forelimb. The importance of ipsilesional hemisphere motor organization for spontaneous contralesional limb use has previously been demonstrated in adult rats (Plowman et al., 2011) and squirrel monkeys (Eisner-Janowicz et al., 2008). However, thus far, the relationship of this behavior to the motor representation in the contralesional hemisphere has received little attention in the literature. The role of the contralesional hemisphere is particularly relevant with respect to targets of inhibitory/facilitatory neuromodulation therapies (Kirton, 2013; Dukelow and Kirton, 2020).

In the single-pellet task, which measures skilled forelimb grasping and retrieval, only motor map size in the ipsilesional hemisphere was correlated with impairment, as measured by the average number of reach attempts required for a successful pellet retrieval. This significant correlation was observed both for the combined sham and stroke groups, as well as for the stroke group alone. The relationship between ipsilesional motor map size and skilled forelimb use is well established (Conner et al., 2003; Plowman et al., 2011; Nishibe et al., 2015; Kim et al., 2018). Interestingly, we also show a positive relationship between the contralesional hemisphere and spontaneous forelimb use in the cylinder task, which has yet to be reported within any preclinical perinatal injury studies.

It is of interest that the motor map size and movement response latencies in both hemispheres were correlated with performance in the cylinder task, whereas only the motor map size in the ipsilesional hemisphere was correlated with performance in the single-pellet task. Likewise, lesion volume was also correlated with performance in the single-pellet task, but not the cylinder task. These results are likely because of different types of motor behavior being tested in the spontaneous and skilled forelimb tasks. While the cylinder task requires a combination of postural, proximal, and distal muscle usage in the context of weight-bearing and shifting (Schallert et al., 2000), the single-pellet task requires precise motor control of distal forelimb musculature (i.e., digits, hand, wrist) (Whishaw, 1996; Farr and Whishaw, 2002). Therefore, the cylinder task may rely on greater input from both hemispheres because of the involvement of both axial and proximal muscles, whereas the single-pellet task is a comparatively more lateralized task. Our results also align with the findings of Jeffers et al. (2020) who recently demonstrated in rats that the degree of poststroke impairment and subsequent recovery varied between spontaneous and skilled forelimb use based on the precise

\section{Contralesional Hemi Map Size Ipsilesional Hemi}
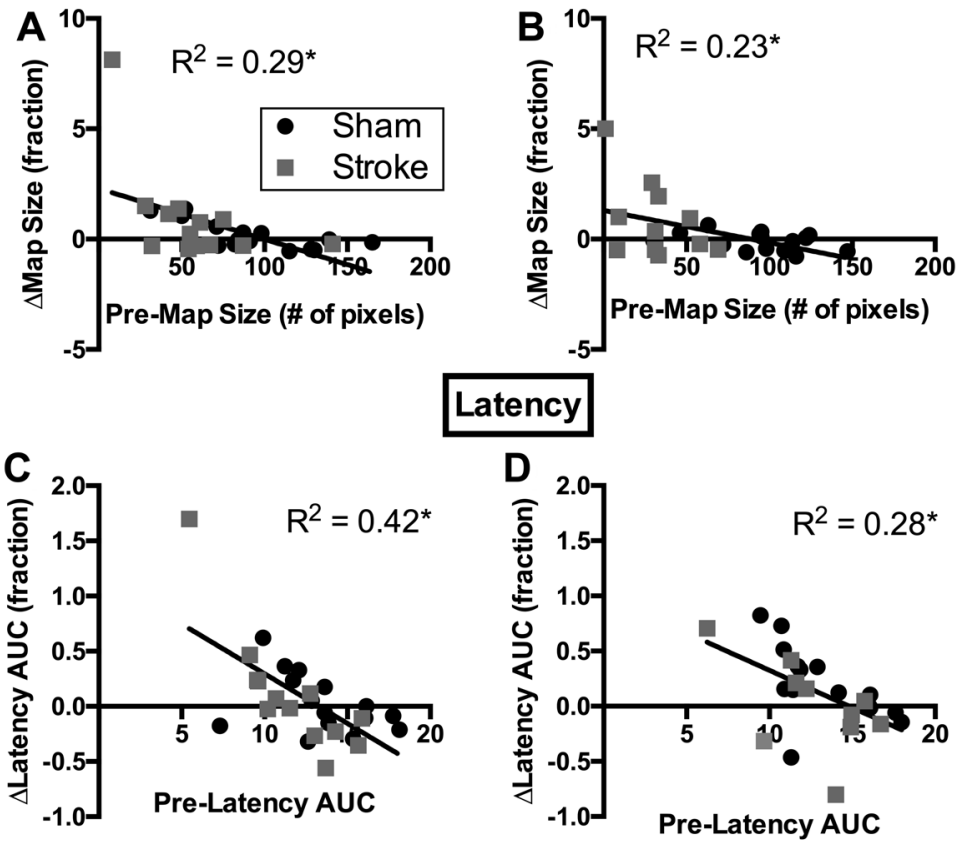

\section{Contralesional Hemi Map Size Ipsilesional Hemi}
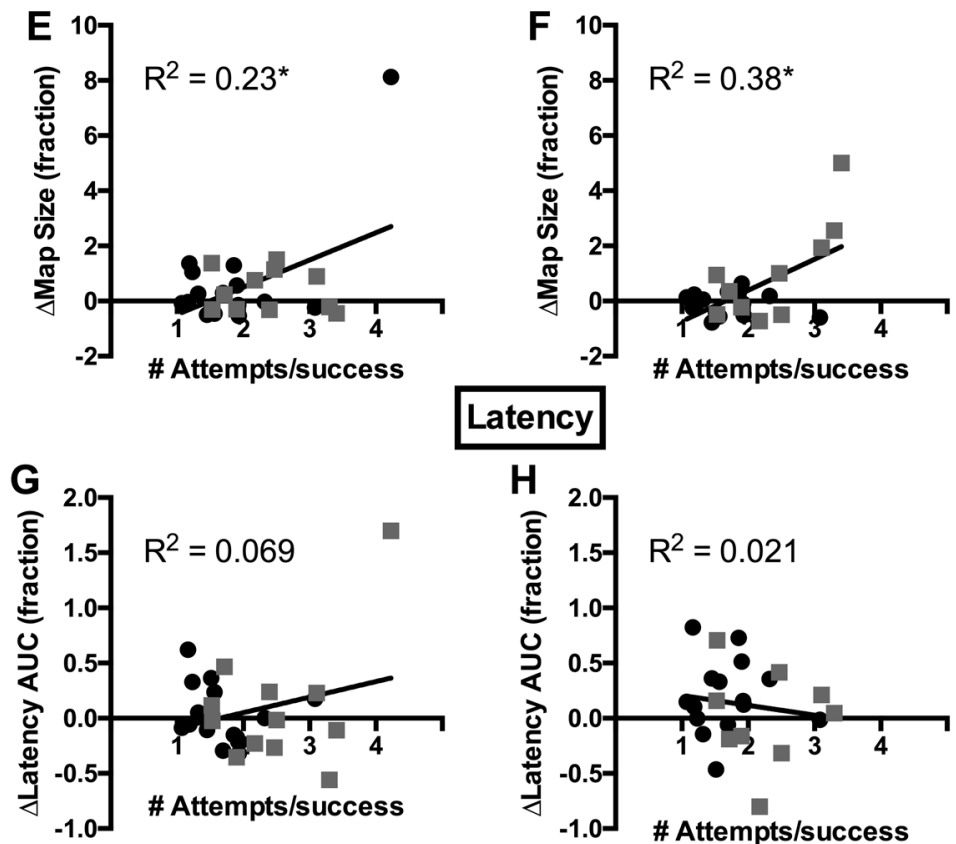

Figure 9. $\quad \boldsymbol{A}, \boldsymbol{B}$, Post-single-pellet change in contralesional forelimb map size was significantly correlated with pre-single-pellet map size in both the contralesional and ipsilesional hemispheres. $\boldsymbol{C}, \boldsymbol{D}$, Post-single-pellet change in contralesional movement latency AUC was also significantly correlated with pre-single-pellet movement latency AUC in both hemispheres. $\boldsymbol{E}, \boldsymbol{F}$, Post-single-pellet change in map size was correlated with number of reaching attempts in both hemispheres. $\boldsymbol{G}, \boldsymbol{H}$, However, change in movement latency AUC was not in either hemisphere. Sham $(n=15)$, stroke $(n=13)$. Animals were excluded because of inability to acquire reaching behaviors. ${ }^{*} p<0.05$.

subregions of the forelimb motor representation that were damaged. Together, these results suggest that the potential beneficial role of both the contralesional and ipsilesional hemispheres is task-specific. This finding has implications for neuromodulation therapies and is particularly relevant to studies using robotic TMS mapping protocols (Grab et al., 2018; Giuffre et al., 2019) 
and repetitive noninvasive TMS therapies (Zewdie et al., 2020) in human pediatric populations.

\section{Skilled motor training may promote enlargement of maps and reduction of motor response latency in those with the smallest pretraining responses}

There is precedent for the capacity to change map size with training in both the ipsilesional (Nudo and Milliken, 1996; Kleim et al., 1998, 2004) and contralesional hemispheres (Pruitt et al., 2016) of adult animals, as well as following perinatal injury in the ipsilesional hemisphere (Williams et al., 2006). However, clinical evidence has shown that perinatal stroke patients with very reduced ipsilesional motor maps show no training-induced expansion with rehabilitation, whereas those with viable maps can (Friel et al., 2016). In our study, following 3 weeks of singlepellet training, there was no overall expansion of motor map size or reductions in motor response latency. As our study did not contain an untrained group of animals, we cannot rule out that our training could have normalized changes to the maps that would have occurred in the absence of training, such as an atrophy of map size. However, given that our assessments were done $\sim 3$ months after injury, with no additional stimuli for inducing behavioral changes, it seems unlikely that such changes would have occurred within the 3 week timeframe of our experiments. Furthermore, our results support the work of others who have also failed to show overall expansions in motor map size using similar training paradigms in rats (Kleim et al., 2004; Young et al., 2012). This could be related to the ability to measure subtle movements. For example, we were not able to distinguish between subtle distal (hand/digit) versus proximal (arm/ shoulder) movements through the accelerometers, and thus could not test whether there was expansion of this specific cortical area. Intensity of training is also a critical modulating factor to trigger changes to descending motor tracts (Friel et al., 2013). In our training protocol, animals were engaged for $10 \mathrm{~min}$ a day for 3 weeks. This may be similar to what some stroke patients receive for their upper limbs in clinical practice (Hayward and Brauer, 2015); however, it may be insufficient to induce neurophysiological changes or promote recovery of function. Previous rodent studies have shown that a critical threshold of rehabilitation intensity should be surpassed to obtain functional benefits in skilled reaching behavior (MacLellan et al., 2011) and that the specific threshold required is modulated by the initial level of impairment and infarct volume of the subject (Jeffers et al., 2018). Given that the size of the perinatal strokes in the present study often impinged on the cortex, corpus callosum, and striatum, it is possible that a high intensity of rehabilitation may be required to induce behavioral change in these animals.

Despite the lack of groupwise changes in motor map size or response latency with skilled training in the present study, we also observed that individual animals with the smallest map sizes and longest response latencies before training were those that showed the most subsequent change in these metrics. This could potentially result from ceiling effects in the measurement, whereby only animals with severe reductions in these metrics have the potential to show large changes, as has been observed for other behavioral outcomes following stroke (Hawe et al., 2019). However, changes in map size were positively correlated with number of reach attempts required to successfully obtain the pellet during skilled reaching. This is a measure that is not necessarily coupled to map size, and theoretically does not have a performance ceiling. Given the correlations between both small map size and severity of skilled reaching impairment to the change observed in map size with motor training, it may be possible that mice with more severe stroke impairments have a greater potential to respond to motor rehabilitation. Similar results have been observed in adult rats, wherein those with more moderate to severe impairment show greater rehabilitation-induced improvements relative to less severely impaired subjects (Jeffers et al., 2018).

\section{Potential limitations of optogenetic mapping}

The present experiment used Thy1-ChR2-YFP mice, as expression of channelrhodopsin within L5 pyramidal neurons has been well characterized in this line (Arenkiel et al., 2007). Compared with previous ICMS motor mapping studies (Kartje-Tillotson et al., 1986; Tennant et al., 2011), we observed motor maps that were somewhat larger and more bilaterally organized; however, these differences could be accounted for by the form of stimulation used. Specifically, blue light optogenetic stimulation does not penetrate deep enough into cortical tissue to directly activate L5 cell bodies (Yizhar et al., 2011). Instead, optogenetic stimulation likely depolarizes the apical tufts of the L5 neurons, leading to orthodromic activation of the cell body (Lim et al., 2013). In contrast, during ICMS stimulation, the local current injection in L5 nondiscriminately activates fibers of passage, leading to antidromic activation of both excitatory and inhibitory neurons (Tehovnik, 1996). Therefore, the activation of local inhibitory neurons during electrical stimulation may prevent movement generation at some stimulation sites (Hussin et al., 2015), whereas the selective, light-based stimulation of excitatory pyramidal neurons likely bypasses this form of inhibitory gating and can evoke movement from a greater number of stimulation sites. An additional methodological difference that may contribute to differences in map size is the fact that our accelerometerbased movement detection may be more sensitive than visual observation of limb movement (as is done during typical ICMS mapping). These differences in stimulation methods, as well as our use of suprathreshold levels of stimulation during mapping, may explain the somewhat larger and bilateral maps that we obtained with optogenetic mapping in the present study.

As optogenetic mapping is becoming increasingly common in longitudinal experiments, it is important to note that several potential limitations of this technique have been characterized or addressed. For example, the use of transcranial chronic windows provides a more stable preparation compared with chronic craniotomy or thin-bone preparations, or acute craniotomies in terminal mapping studies. Bone manipulation in chronic preparations often results in gradual bone regrowth or inflammation that compromises the clarity of the chronic window and the health of the underlying brain tissue (Xu et al., 2007). Surface blood vessels may also pose a problem for both ICMS and optogenetic-based mapping. To minimize injury during ICMS, surface vessels are avoided by slightly shifting an electrode penetration site when the predetermined site falls on a visible surface vessel. In contrast, during optogenetic mapping, the regular spacing of stimulation sites is maintained, even when it falls on a surface vessel, as vessel architecture does not present a significant artifact to stimulation power (Ayling et al., 2009).

Beyond the differences between classical ICMS and optogenetic techniques used in the present study, our results allow us to preliminarily address the utility of motor map size and response latency as biomarkers of perinatal stroke. Originally, we defined several experimental goals to begin to answer this question: (1) Are motor maps correlated with adult impairment? - Yes, we observed that loss of motor maps because of perinatal stroke 
resulted in an increased number of reach attempts required to retrieve pellets in a skilled reaching task during adulthood. (2) Can the maps be altered by skilled motor training? - Perhaps not, the 3 week, 10 min per day training paradigm used in the present study did not result in changes to motor maps. However, we cannot rule out that our training may have normalized potential loss of maps, or that a higher-intensity paradigm could potentially expand map sizes. (3) Does the initial behavioral impairment modulate the degree to which these metrics can change in response to training? - Yes, mice with the greatest impairments also displayed the greatest increase in motor map size throughout the course of their training. These results indicate that motor map size warrants further investigation for its potential as a biomarker in perinatal stroke.

We believe that to serve as a good biomarker for perinatal stroke, motor maps would need to be able to fulfill at least one of three potential clinical functions: (1) early prediction of how an individual's course of impairment will either progress or recover; (2) aid in decision-making about the course of treatments that the individual should receive; or (3) enable stratification of patient groups, especially in the context of refining selection criteria for clinical trials of prospective therapies. Although the present study does not fulfill any of these functions, it does provide a good justification for proceeding to investigate such functions in future experiments. It may be possible to use our noninvasive mapping techniques to obtain motor maps earlier in life and observe the evolution of motor maps and impairments with aging. These data could be used to develop predictive models and profiles of individuals that would be expected to have good or bad outcomes, as is possible using TMS measures in adult human stroke (Stinear et al., 2017). Experimental therapeutic or neuromodulatory procedures could be varied based on these stratification profiles to aid development of optimized, and perhaps even individualized, treatment strategies.

In conclusion, the present experiment demonstrates that both motor map size and movement latency have qualities that may make them suitable as potential biomarkers of impairment and recovery from perinatal stroke. However, motor map size may be the preferable measure. Both movement latency and motor map size were negatively impacted by stroke and correlated with impairment in spontaneous limb use. However, motor map size was additionally correlated with lesion volume and average number of reach attempts required to perform a successful skilled reach, as well as a positive association between degree of initial impairment and training-induced change. This implies that the larger the lesion volume, the greater the reduction in map size and greater the number of reach attempts required to successfully complete a pellet retrieval. Those with the greatest reductions in map size may have the most potential for subsequent positive change. Future studies of perinatal stroke rehabilitation and neuromodulation should include measures of motor map size and movement latency to unravel the neurophysiological changes that are occurring in response to treatment.

\section{References}

Anenberg E, Arstikaitis P, Niitsu Y, Harrison TC, Boyd JD, Hilton BJ, Tetzlaff W, Murphy TH (2014) Ministrokes in channelrhodopsin-2 transgenic mice reveal widespread deficits in motor output despite maintenance of cortical neuronal excitability. J Neurosci 34:1094-1104.

Arenkiel BR, Peca J, Davison IG, Feliciano C, Deisseroth K, Augustine GJ, Ehlers MD, Feng G (2007) In vivo light-induced activation of neural circuitry in transgenic mice expressing channelrhodopsin-2. Neuron 54:205-218.
Ayling OG, Harrison TC, Boyd JD, Goroshkov A, Murphy TH (2009) Automated light-based mapping of motor cortex by photoactivation of channelrhodopsin-2 transgenic mice. Nat Methods 6:219-224.

Bell KJ, Õunpuu S, DeLuca PA, Romness MJ (2002) Natural progression of gait in children with cerebral palsy. J Pediatr Orthop 22:677-682.

Bernhardt J, Borschmann K, Boyd L, Carmichael ST, Corbett D, Cramer SC, Hoffmann T, Kwakkel G, Savitz SI, Saposnik G, Walker M, Ward N (2016) Moving rehabilitation research forward: developing consensus statements for rehabilitation and recovery research. Int J Stroke 11:454458.

Boonzaier J, Tilborg GV, Neggers SF, Dijkhuizen RM (2018) Noninvasive brain stimulation to enhance functional recovery after stroke: studies in animal models. Neurorehabil Neural Repair 32:927-940.

Bouet V, Boulouard M, Toutain J, Divoux D, Bernaudin M, Schumann-Bard $P$, Freret T (2009) The adhesive removal test: a sensitive method to assess sensorimotor deficits in mice. Nat Protoc 4:1560-1564.

Boyd LA, Hayward KS, Ward NS, Stinear CM, Rosso C, Fisher RJ, Carter AR, Leff AP, Copland DA, Carey LM, Cohen LG, Basso DM, Maguire JM, Cramer SC (2017) Biomarkers of stroke recovery: consensus-based core recommendations from the Stroke Recovery and Rehabilitation Roundtable. Int J Stroke 12:480-493.

Brima T, Mikulecká A, Otáhal J (2013) Impacts of perinatal induced photothrombotic stroke on sensorimotor performance in adult rats. Physiol Res 62:85-94.

Brus-Ramer M, Carmel JB, Martin JH (2009) Motor cortex bilateral motor representation depends on subcortical and interhemispheric interactions. J Neurosci 29:6196-6206.

Carlson HL, MacMaster FP, Harris AD, Kirton A (2017) Spectroscopic biomarkers of motor cortex developmental plasticity in hemiparetic children after perinatal stroke. Hum Brain Mapp 38:1574-1587.

Carr LJ, Harrison LM, Evans AL, Stephens JA (1993) Patterns of central motor reorganization in hemiplegic cerebral palsy. Brain 116:1223-1247.

Castro J (1975) lpsilateral corticospinal projections after large lesions of the cerebral hemisphere in neonatal rats. Exp Neurol 46:1-8.

Cavarsan CF, Gorassini MA, Quinlan K (2019) Animal models of developmental motor disorders: parallels to human motor dysfunction in cerebral palsy. J Neurophysiol 122:1238-1253.

Clancy B, Darlington RB, Finlay BL (2001) Translating developmental time across mammalian species. Neuroscience 105:7-17.

Conner JM, Culberson A, Packowski C, Chiba AA, Tuszynski MH, Diego S (2003) Lesions of the basal forebrain cholinergic system impair task acquisition and abolish cortical plasticity associated with motor skill learning. Neuron 38:819-829.

Dewar D, Underhill SM, Goldberg MP (2003) Oligodendrocytes and ischemic brain injury. J Cereb Blood Flow Metab 23:263-274.

Dukelow S, Kirton A (2020) Enhancing stroke recovery across the life span with noninvasive neurostimulation. J Clin Neurophysiol 37:150-163.

Dunbar M, Mineyko A, Hill M, Hodge J, Floer A, Kirton A (2020) Population based birth prevalence of disease-specific perinatal stroke. Pediatrics 146:e2020013201.

Eisner-Janowicz I, Barbay S, Hoover E, Stowe AM, Frost SB, Plautz EJ, Nudo RJ (2008) Early and late changes in the distal forelimb representation of the supplementary motor area after injury to frontal motor areas in the squirrel monkey. J Neurophysiol 100:1498-1512.

Eyre JA, Smith M, Dabydeen L, Clowry GJ, Petacchi E, Battini R, Guzzetta A, Cioni G (2007) Is hemiplegic cerebral palsy equivalent to amblyopia of the corticospinal system? Ann Neurol 62:493-503.

Eyre JA, Taylor JP, Villagra F, Smith M, Miller S (2001) Evidence of activitydependent withdrawal of corticospinal projections during human development. Neurology 57:1543-1554.

Farr TD, Whishaw IQ (2002) Quantitative and qualitative impairments in skilled reaching in the mouse (Mus musculus) after a focal motor cortex stroke. Stroke 33:1869-1875.

Ferriero DM (2004) Neonatal brain injury. N Engl J Med 351:1985-1995.

Fleming SM, Salcedo J, Fernagut PO, Rockenstein E, Masliah E, Levine MS, Chesselet MF (2004) Early and progressive sensorimotor anomalies in mice overexpressing wild-type human $\alpha$-synuclein. J Neurosci 24:94349440.

Friel KM, Chakrabarty S, Martin JH (2013) Pathophysiological mechanisms of impaired limb use and repair strategies for motor systems after unilateral injury of the developing brain. Dev Med Child Neurol 55:27-31. 
Friel KM, Kuo H, Fuller J, Ferre CL, Brandão M, Carmel JB, Bleyenheuft Y, Gowatsky JL, Stanford AD, Rowny SB, Luber B, Bassi B, Murphy DL, Lisanby SH, Gordon AM (2016) Skilled bimanual training drives motor cortex plasticity in children with unilateral cerebral palsy. Neurorehabil Neural Repair 30:834-844.

Gennaro M, Mattiello A, Pizzorusso T (2019) Rodent models of developmental ischemic stroke for translational research: strengths and weaknesses. Neural Plast 2019:5089321.

Gharbawie OA, Gonzalez CL, Williams PT, Kleim JA, Whishaw IQ (2005) Middle cerebral artery (MCA) stroke produces dysfunction in adjacent motor cortex as detected by intracortical microstimulation in rats. Neuroscience 130:601-610.

Gianino S, Stein SA, Li H, Lu X, Biesiada E, Ulas J, Xu XM (1999) Postnatal growth of corticospinal axons in the spinal cord of developing mice. Brain Res Dev Brain Res 112:189-204.

Giuffre A, Cole L, Kuo HC, Kirton A, Zewdie E, Carlson HL, Grab J (2019) Non-invasive modulation and robotic mapping of motor cortex in the developing brain. J Vis Exp 149:1-12.

Grab JG, Zewdie E, Carlson HL, Kuo H, Ciechanski P, Hodge J, Giuffre A, Kirton A (2018) Robotic TMS mapping of motor cortex in the developing brain. J Neurosci Methods 309:41-54.

Hagberg H, Bona E, Gilland E, Puka-Sundvall M (1997) Hypoxia-ischaemia model in the 7-day-old rat: possibilities and shortcomings. Acta Paediatr Suppl 422:85-88.

Hampton TG, Stasko MR, Kale A, Amende I, Costa ACS (2004) Gait dynamics in trisomic mice: quantitative neurological traits of Down syndrome. Physiol Behav 82:381-389.

Harrison TC, Ayling OG, Murphy TH (2012) Distinct cortical circuit mechanisms for complex forelimb movement and motor map topography. Neuron 74:397-409.

Hawe RL, Scott SH, Dukelow SP (2019) Taking proportional out of stroke recovery. Stroke 50:204-211.

Hayward KS, Brauer SG (2015) Dose of arm activity training during acute and subacute rehabilitation post stroke: a systematic review of the literature. Clin Rehabil 29:1234-1243.

Heald A, Bates D, Cartlidge NE, French JM, Miller S (1993) Longitudinal study of central motor conduction time following stroke: 1 . Natural history of central motor conduction. Brain 116:1355-1370.

Hicks SP, Amato CJ (1975) Motor-sensory cortex: corticospinal system and developing locomotion and placing in rats. Am J Anat 143:1-41.

Hilderley AJ, Metzler MJ, Kirton A (2019) Noninvasive neuromodulation to promote motor skill gains after perinatal stroke. Stroke 50:233-239.

Hira R, Honkura N, Noguchi J, Maruyama Y, Augustine GJ, Kasai H, Matsuzaki M (2009) Transcranial optogenetic stimulation for functional mapping of the motor cortex. J Neurosci Methods 179:258-263.

Hsu JY, Stein SA, Xu XM (2006) Development of the corticospinal tract in the mouse spinal cord: a quantitative ultrastructural analysis. Brain Res 1084:16-27.

Hussin AT, Boychuk JA, Brown AR, Pittman QJ, Campbell Teskey G (2015) Intracortical microstimulation (ICMS) activates motor cortex layer 5 pyramidal neurons mainly transsynaptically. Brain Stimul 8:742-750.

Jaspers E, Byblow WD, Feys H, Wenderoth N (2016) The corticospinal tract: a biomarker to categorize upper limb functional potential in unilateral cerebral palsy. Front Pediatr 3:1-10.

Jeffers MS, Karthikeyan S, Gomez-Smith M, Gasinzigwa S, Achenbach J, Feiten A, Corbett D (2018) Does stroke rehabilitation really matter? Part B: an algorithm for prescribing an effective intensity of rehabilitation. Neurorehabil Neural Repair 32:73-83.

Jeffers MS, Touvykine B, Ripley A, Lahey G, Carter A, Dancause N, Corbett D (2020) Post-stroke impairment and recovery are predicted by task-specific regionalization of injury. J Neurosci 40:6082-6097.

Joosten EA, Gribnau AA, Dederen PJ (1987) An anterograde tracer study of the developing corticospinal tract in the rat: three components. Dev Brain Res 36:121-130.

Kartje-Tillotson G, Neafsey EJ, Castro AJ (1985) Electrophysiological analysis of motor cortical plasticity after cortical lesions in newborn rats. Brain Res 332:103-111.

Kartje-Tillotson G, Neafsey EJ, Castro AJ (1986) Topography of corticopontine remodelling after cortical lesions in newborn rats. J Comp Neurol 250:206-214.

Kim SY, Allred RP, De Adkins AL, Tennant KA, Donlan NA, Kleim JA, Jones TA (2015) Experience with the "good" limb induces aberrant synaptic plasticity in the perilesion cortex after stroke. J Neurosci 35:8604-8610.

Kim SY, Hsu JE, Husbands LC, Kleim JA, Jones TA (2018) Coordinated plasticity of synapses and astrocytes underlies practice-driven functional vicariation in peri-infarct motor cortex. J Neurosci 38:93-107.

Kirton A (2013) Modeling developmental plasticity after perinatal stroke: defining central therapeutic targets in cerebral palsy. Pediatr Neurol 48:81-94

Kleim JA, Barbay S, Nudo RJ, Jeffrey A, Barbay S, Func RJ (1998) Functional reorganization of the rat motor cortex following motor skill learning. J Neurophysiol 80:3321-3325.

Kleim JA, Hogg TM, Vandenberg PM, Cooper NR, Bruneau R, Remple M (2004) Cortical synaptogenesis and motor map reorganization occur during late, but not early, phase of motor skill learning. J Neurosci 24:628633.

Kolb B, Holmes C (1983) Neonatal motor cortex lesions in the rat: absence of sparing of motor behaviors and impaired spatial learning concurrent with abnormal cerebral morphogenesis. Behav Neurosci 97:697-709.

Koski L, Mernar TJ, Dobkin BH (2004) Immediate and long-term changes in corticomotor output in response to rehabilitation: correlation with functional improvements in chronic stroke. Neurorehabil Neural Repair $18: 230-249$

Kowalski JL, Nemanich ST, Nawshin T, Chen M, Peyton C, Zorn E, Hickey M, Rao R, Georgieff M, Rudser K, Gillick BT (2019) Motor evoked potentials as potential biomarkers of early atypical corticospinal tract development in infants with perinatal stroke. J Clin Med 8:1208.

Lim DH, LeDue J, Mohajerani MH, Vanni MP, Murphy TH (2013) Optogenetic approaches for functional mouse brain mapping. Front Neurosci 7:1-15.

MacLellan CL, Keough MB, Granter-Button S, Chernenko GA, Butt S, Corbett D (2011) A critical threshold of rehabilitation involving brainderived neurotrophic factor is required for poststroke recovery. Neurorehabil Neural Repair 25:740-748.

Maxwell KA, Dyck H (2005) Induction of reproducible focal ischemic lesions in neonatal mice by photothrombosis. Dev Neurosci 27:121-126.

McQuillen PS, Ferriero DM (2004) Selective vulnerability in the developing central nervous system. Pediatr Neurol 30:227-235.

Monfils MH, Driscoll I, Vandenberg PM, Thomas NJ, Danka D, Kleim JA, Kolb B (2005) Basic fibroblast growth factor stimulates functional recovery after neonatal lesions of motor cortex in rats. Neuroscience 134:1-8.

Monfils MH, Driscoll I, Vavrek R, Kolb B, Fouad K (2008) FGF-2-induced functional improvement from neonatal motor cortex injury via corticospinal projections. Exp Brain Res 185:453-460.

Nishibe M, Urban IIE, Barbay S, Nudo RJ (2015) Rehabilitative training promotes rapid motor recovery but delayed motor map reorganization in a rat cortical ischemic infarct model. Neurorehabil Neural Repair 29:472482.

Nudo RJ, Milliken GW (1996) Reorganization of movement representations in primary motor cortex following focal ischemic infarcts in adult squirrel monkeys. J Neurophysiol 75:2144-2149.

Plowman EK, Thomas NJ, Kleim JA (2011) Striatal dopamine depletion induces forelimb motor impairments and disrupts forelimb movement representations within the motor cortex. J Parkinsons Dis 1:93-100.

Pruitt DT, Schmid AN, Danaphongse TT, Flanagan KE, Morrison RA, Kilgard MP, Rennaker RL, Hays SA (2016) Forelimb training drives transient map reorganization in ipsilateral motor cortex. Behav Brain Res 313:10-16.

Rethlefsen SA, Kay RM (2013) Transverse plane gait problems in children with cerebral palsy. J Pediatr Orthop 33:422-430.

Riddell M, Kuo H, Zewdie E, Kirton A (2019) Mirror movements in children with unilateral cerebral palsy due to perinatal stroke: clinical correlates of plasticity reorganization. Dev Med Child Neurol 61:943-949.

Schallert T, Fleming SM, Leasure JL, Tillerson JL, Bland ST (2000) CNS plasticity and assessment of forelimb sensorimotor outcome in unilateral rat models of stroke, cortical ablation, parkinsonism and spinal cord injury. Neuropharmacology 39:777-787.

Schallert T, Woodlee MT, Fleming SM (2002) Disentangling multiple types of recovery from brain injury. In: Pharmacology of cerebral ischemia (Krieglstein J, Klumpp S, eds), pp 201-216. Stuttgart, Germany: Medpharm Scientific.

Silasi G, Boyd JD, Ledue J, Murphy TH, Hirase H, Science RB (2013) Improved methods for chronic light-based motor mapping in mice: 
automated movement tracking with accelerometers, and chronic EEG recording in a bilateral thin-skull preparation. Front Neural Circuits 7:110

Silasi G, Xiao D, Vanni MP, Chen AC, Murphy TH (2016) Intact skull chronic windows for mesoscopic wide-field imaging in awake mice. J Neurosci Methods 267:141-149.

Staudt M (2007) (Re-)organization of the developing human brain following periventricular white matter lesions. Neurosci Biobehav Rev 31:11501156.

Stinear CM, Byblow WD, Ackerley SJ, Smith MC, Borges VM, Barber PA (2017) PREP2: a biomarker-based algorithm for predicting upper limb function after stroke. Ann Clin Transl Neurol 4:811-820.

Stinear CM, Smith MC, Byblow WD (2019) Prediction tools for stroke rehabilitation. Stroke 50:3314-3322.

Tehovnik EJ (1996) Electrical stimulation of neural tissue to evoke behavioral responses. J Neurosci Methods 65:1-17.

Tennant KA, Adkins DL, Donlan NA, Asay AL, Thomas N, Kleim JA, Jones TA (2011) The organization of the forelimb representation of the C57BL/ 6 mouse motor cortex as defined by intracortical microstimulation and cytoarchitecture. Cereb Cortex 21:865-876.

Terashima T (1995) Anatomy, development and lesion-induced plasticity of rodent corticospinal tract. Neurosci Res 22:139-161.

Touvykine B, Mansoori BK, Jean-Charles L, Deffeyes J, Quessy S, Dancause N (2016) The effect of lesion size on the organization of the ipsilesional and contralesional motor cortex. Neurorehabil Neural Repair 30:280292.

Touvykine B, Elgbeili G, Quessy S, Dancause N (2020) Interhemispheric modulations of motor outputs by the rostral and caudal forelimb areas in the rat. J Neurophysiol 123:1355-1368.

Turton A, Wroe S, Trepte N, Fraser C, Lemon RN (1996) Contralateral and ipsilateral EMG responses to transcranial magnetic stimulation during recovery of arm and hand function after stroke. Electroencephalogr Clin Neurophysiol 101:316-328.

Vahabzadeh-Hagh AM, Muller PA, Gersner R, Zangen A, Rotenberg A (2012) Translational neuromodulation: approximating human transcranial magnetic stimulation protocols in rats. Neuromodulation 15:296305.
Vandermeeren Y, Bastings E, Fadiga L, Olivier E (2003) Long-latency motor evoked potentials in congenital hemiplegia. Clin Neurophysiol 114:18081818.

Wang X, Wang Y (2012) Gait analysis of children with spastic hemiplegic cerebral palsy. Neural Regen Res 7:1578-1584.

Whishaw IQ (1996) An endpoint, descriptive, and kinematic comparison of skilled reaching in mice (Mus musculus) with rats (Rattus norvegicus). Behav Brain Res 78:101-111.

Whishaw IQ, Kolb B (1988) Sparing of skilled forelimb reaching and corticospinal projections after neonatal motor cortex removal or hemidecortication in the rat: support for the Kennard doctrine. Brain Res 451:97-114.

Williams PT, Gharbawie OA, Kolb B, Kleim JA (2006) Experience-dependent amelioration of motor impairments in adulthood following neonatal medial frontal cortex injury in rats is accompanied by motor map expansion. Neuroscience 141:1315-1326.

Wren TA, Rethlefsen S, Kay RM (2005) Prevalence of specific gait abnormalities in children with cerebral palsy: influence of cerebral palsy subtype, age, and previous surgery. J Pediatr Orthop 25:79-83.

Xu HT, Pan F, Yang G, Gan WB (2007) Choice of cranial window type for in vivo imaging affects dendritic spine turnover in the cortex. Nat Neurosci 10:549-551.

Yizhar O, Fenno LE, Davidson TJ, Mogri M, Deisseroth K (2011) Optogenetics in neural systems. Neuron 71:9-34.

Young NA, Vuong J, Campbell Teskey G (2012) Development of motor maps in rats and their modulation by experience. J Neurophysiol 108:1309-1317.

Zewdie E, Damji O, Ciechanski P, Seeger T, Kirton A (2017) Contralesional corticomotor neurophysiology in hemiparetic children with perinatal stroke: developmental plasticity and clinical function. Neurorehabil Neural Repair 31:261-271.

Zewdie E, Ciechanski P, Kuo HC, Giuffre A, Kahl C, King R, Cole L, Godfrey H, Seeger T, Swansburg R, Damji O, Rajapakse T, Hodge J, Nelson S, Selby B, Gan L, Jadavji Z, Larson JR, MacMaster F, Yang JF, et al. (2020) Safety and tolerability of transcranial magnetic and direct current stimulation in children: prospective single center evidence from 3.5 million stimulations. Brain Stimul 13:565-575.

Zwick EB, Saraph V, Linhart WE, Steinwender G (2001) Propulsive function during gait in diplegic children: evaluation after surgery for gait improvement. J Pediatr Orthop 10:226-233. 